\title{
Classifying the Metrics for Which Geodesic Flow on the Group $S O(n)$ is Algebraically Completely Integrable
}

\author{
Lesfari Ahmed ${ }^{1 *}$
}

\begin{abstract}
The aim of this paper is to demonstrate the rich interaction between the Kowalewski-Painleve analysis, the properties of algebraic completely integrable (a.c.i.) systems, the geometry of its Laurent series solutions, and the theory of Abelian varieties. We study the classification of metrics for which geodesic flow on the group $S O(n)$ is a.c.i. For $n=3$, the geodesic flow on $S O(3)$ is always a.c.i., and can be regarded as the Euler rigid body motion. For $n=4$, in the Adler-van Moerbeke's classification of metrics for which geodesic flow on $S O(4)$ is a.c.i., three cases come up; two are linearly equivalent to the Clebsch and Lyapunov-Steklov cases of rigid body motion in a perfect fluid, and there is a third new case namely the Kostant-Kirillov Hamiltonian flow on the dual of $s o(4)$. Finally, as was shown by Haine, for $n \geq 5$ Manakov's metrics are the only left invariant diagonal metrics on $S O(n)$ for which the geodesic flow is a.c.i.
\end{abstract}

Keywords: Jacobians varieties, Prym varieties, Integrable systems, Topological structure of phase space, Methods of integration.

2010 AMS: Primary 37J35, Secondary 14H40, 70H06, 14H70

${ }^{1}$ Department of Mathematics, Faculty of Sciences, University of Chouaïb Doukkali, B.P. 20, 24000 El Jadida, Morocco, ORCID:

0000-0001-6213-4301

*Corresponding author: lesfariahmed@yahoo.fr

Received: 21 November 2019, Accepted: 5 February 2020, Available online: 25 March 2020

\section{Introduction}

A dynamical system is algebraic completely integrable in the sense of Adler-van Moerbeke [3, 6] if it can be linearized on a complex algebraic torus (Abelian variety). The invariants (often called first integrals or constants) of the motion are polynomials and the phase space coordinates, or some algebraic functions of these, restricted to a complex invariant variety defined by putting these invariants equals to generic constants are meromorphic functions on an Abelian variety. These manifolds are described explicitly as being affine part of complex algebraic tori and the flows (run with complex time) generated by the constants of the motion can be solved by quadrature, that is to say their solutions can be expressed in terms of Abelian integrals.

Consider the group $S O(n)$ and its Lie algebra $s o(n)$ paired with itself, via the customary inner product

$$
\langle X, Y\rangle=-\frac{1}{2} \operatorname{tr}(X . Y), \quad X, Y \in \operatorname{so}(n)
$$

A left invariant metric on $S O(n)$ is defined by a non-singular symmetric linear map

$$
\Lambda: \operatorname{so}(n) \longrightarrow \operatorname{so}(n), \quad X \longmapsto \Lambda . X
$$


and by the following inner product; given two vectors $g X$ and $g Y$ in the tangent space $S O(n)$ at the point $g \in S O(n)$,

$$
\langle g X, g Y\rangle=\left\langle X, \Lambda^{-1} . Y\right\rangle .
$$

The question of classifying the metrics for which geodesic flow on $S O(n)$ is algebraically completely integrable is difficult. For $S O(3)$, we know that the Euler rigid body motion [11] can be regarded as geodesic flow on $S O(3)$ and this problem is always algebraically completely integrable. For $S O(4)$, Adler and van Moerbeke [4] have shown that the geodesic flow on $S O(4)$ for the metric defined by the above quadratic form is algebraically completely integrable in the three cases described in the section 5 and these are the only ones that exist. In addition, these three cases come up: two are linearly equivalent to cases of rigid body motion in a perfect fluid studied there is a long time, respectively by Clebsch and Lyapunov-Steklov, an there is a third new case namely the Kostant-Kirillov Hamiltonian flow on the dual of $s o(4)$. For $n \geq 5$, as was shown by Haine [14], Manakov's metrics are the only left invariant diagonal metrics on $S O(n)$ for which the geodesic flow is algebraically completely integrable.

The paper is organized as follows. In Section 2, we explain the notion of algebraically completely integrable systems. Section 3 deals with geodesic flow on $S O(3)$ and Euler rigid body motion. In this section, we show that the Euler rigid body motion is always algebraically completely integrable which can be regarded as geodesic flow on $S O(3)$, and the integration of the equations is done by means of elliptic Jacobi functions. In section 4, we briefly recall some results concerning the Clebsch and Lyapunov-Steklov cases of a solid in an ideal fluid. Section 5 deals with the Adler-van Moerbeke classification of algebraic integrable geodesic flow on the group $S O(4)$. This classification concerns Manakov geodesic flow on the group $S O(4)$ which is linearly equivalent to Clebsch rigid body motion in a perfect fluid, geodesic flow on the group $S O(4)$ which is linearly equivalent to Lyapunov-Steklov rigid body motion in a perfect fluid and geodesic flow on $S O(4)$ with a quartic invariant. Section 6 deals with the algebraic complete integrability of geodesic flow on $S O(n)$ for $n \geq 5$.

\section{Algebraic complete integrability}

The definition of the algebraic complete integrability of a Hamiltonian system varies according to the literature and is usually found (with some minor variants) in any modern text on integrable systems. The integrable systems that we will deal with here are complex integrable systems on an affine space $\mathbb{C}^{m}$, the algebra that we consider is just that of the polynomial functions and we focus on algebraic complete integrability in the sense of Adler-van Moerbeke. We will work with complexes instead of real ones. Concepts such as: Liouville integrability, involution, commutativity of vector fields and so on, can be defined as in the real case. On the other hand difficulties arise : we know that there are no compact holomorphic submanifolds in the complex space $\mathbb{C}^{m}$ (maximum principle), therefore the complex tori that we can get in Arnold Liouville's theorem are not compact. The difficult problem of the compactification of invariant varieties therefore arises. In addition, the solutions of the system in question are not uniform (single-valued). First, we will recall some results, define and explain the concept of algebraic complete integrability of Hamiltonian systems in general (although for the problems studied in this paper, we will be concerned by the affine space $\mathbb{C}^{m}$ ).

Consider a Hamiltonian completely integrable system

$$
X_{H}: \dot{z}=J \frac{\partial H}{\partial z} \equiv f(z), z \in \mathbb{R}^{m}, \quad m=2 n+k, \quad J(z) \text { polynomial in } z
$$

with $n+k$ functionally independent invariants $H_{1}, \ldots, H_{n+k}$ of which $k$ invariants (Casimir functions) lead to zero vector fields

$$
J \frac{\partial H_{n+j}}{\partial z}(z)=0, \quad 1 \leq j \leq k,
$$

the $n=(m-k) / 2$ remaining ones are in involution (i.e., $\left.\left\{H_{i}, H_{j}\right\}=0\right)$, which give rise to $n$ commuting vector fields. According to the Arnold-Liouville theorem [7], if the invariant manifolds

$$
\bigcap_{i=1}^{n+k}\left\{z \in \mathbb{R}^{m}: H_{i}(z)=c_{i}\right\},
$$

are compact, then for most values of $c_{i} \in \mathbb{R}$, their connected components are diffeomorphic to real tori $\mathbb{R}^{n} /$ Lattice and the flows $g_{t}^{X_{1}}(x), \ldots, g_{t}^{X_{n}}(x)$ defined by the vector fields $X_{H_{1}}, \ldots, X_{H_{n}}$, are straight-line motions on these tori.

Consider now $z \in \mathbb{C}^{m}$ and $t \in \mathbb{C}$. Let $\Delta \subset \mathbb{C}^{m}$ be a non-empty Zariski open set. By the functional independence of the first integrals, the map (momentum mapping)

$$
\varphi \equiv\left(H_{1}, \ldots, H_{n+k}\right): \mathbb{C}^{m} \longrightarrow \mathbb{C}^{n+k}
$$


is a generic submersion (i.e., $d H_{1}(z), \ldots, d H_{n+k}(z)$ are linearly independent) on $\Delta$. Let

$$
\Omega=\left\{c=\left(c_{i}\right) \in \mathbb{C}^{n+k}: \exists z \in \varphi^{-1}(c) \text { with } d H_{1}(z) \wedge \ldots \wedge d H_{n+k}(z)=0\right\},
$$

be the set of critical values of the map $\varphi$, i.e., $\Omega=\varphi\left(\mathbb{C}^{m} \backslash \Delta\right)$ and denote by $\bar{\Omega}$ the Zariski closure of $\Omega$ in $\mathbb{C}^{n+k}$.

Proposition 2.1. The set defined by

$$
\Gamma=\left\{z \in \mathbb{C}^{m}: \varphi(z) \in \mathbb{C}^{n+k} \backslash \bar{\Omega}\right\}
$$

is everywhere dense in $\mathbb{C}^{m}$ for the usual topology.

Proof. Indeed, it suffices to show that the set $\Gamma=\varphi^{-1}\left(\mathbb{C}^{n+k} \backslash \bar{\Omega}\right)$, is a non-empty Zariski open set in $\mathbb{C}^{m}$. Since a polynomial mapping between affine algebraic sets is continuous for the Zariski topology, then the above set is indeed a Zariski open set in $\mathbb{C}^{m}$ and it is nonempty. Suppose this one is empty, that is $\varphi\left(\mathbb{C}^{m}\right) \subset \bar{\Omega}$. Since the map $\varphi$ is submersive on a non-empty open set of Zariski $\Delta \subset \mathbb{C}^{m}$, then $\varphi(\Delta)$ is open in $\mathbb{C}^{n+k}$. By Sard's theorem for varieties [33], $\mathbb{C}^{n+k} \backslash \bar{\Omega}$ is a non-empty Zariski open set and therefore everywhere dense for the usual topology in $\mathbb{C}^{n+k}$. So $\varphi(\Delta) \cap\left(\mathbb{C}^{n+k} \backslash \bar{\Omega}\right) \neq \emptyset$, which is absurd. This completes the proof.

Let $M_{c}$ be the complex affine variety defined by

$$
M_{c} \equiv \varphi^{-1}(c)=\bigcap_{i=1}^{n+k}\left\{z \in \mathbb{C}^{m}: H_{i}(z)=c_{i}\right\}
$$

for all $c \equiv\left(c_{1}, \ldots, c_{n+k}\right) \in \mathbb{C}^{n+k} \backslash \bar{\Omega}$, the fiber $M_{c}$ is smooth.

Definition 2.2. The system (2.1) is algebraic complete integrable (a.c.i.) in the sense of Adler-van Moerbeke with Abelian functions $z_{i}[3,6,26]$ when, for every $c \in \mathbb{C}^{n+k} \backslash \bar{\Omega}$, the fiber $M_{c}$ (2.2) is the affine part of an Abelian variety (complex algebraic torus)

$$
\widetilde{M}_{c}=T^{n} \simeq \mathbb{C}^{n} / L_{c}, \quad\left(L_{c} \text { a lattice in } \mathbb{C}^{n}\right)
$$

and moreover, the flows $g_{X_{i}}^{t}(z), z \in M_{c}, t \in \mathbb{C}$, defined by the vector fields $X_{H_{i}}, 1 \leq i \leq n$, are straight line on $T^{n}$, i.e.,

$$
\left[g_{X_{i}}^{t}(z)\right]_{j}=f_{j}\left(p+t\left(k_{1}^{i}, \ldots, k_{n}^{i}\right)\right)
$$

where $f_{j}\left(t_{1}, \ldots, t_{n}\right)$ are Abelian functions on $T^{n}, f_{j}(p)=z_{j}, 1 \leq j \leq m$.

We will be concerned with a.c.i. systems that are irreducible i.e., when the generic Abelian variety is irreducible (that is, it does not contain a subtorus). The following remark is intended to present interrelated definitions, all involving a.c.i. systems. For comments on definitions, see [38].

Remark 2.3. 1) [34] Let $H$ be a smooth function on a $2 n$-dimensional symplectic manifold (M, $\omega)$. The Hamiltonian system defined by the vector field $X_{H}$ is a.c.i., if there exists a smooth algebraic variety $\mathscr{M}$, a co-symplectic structure $\widetilde{\omega}$ which restricts to $\omega$ along $M$, i.e., $\widetilde{\omega} \in \Lambda^{2} T_{\mathscr{M}}$ and a morphism $h: \mathscr{M} \longrightarrow \mathscr{U}$ where $\mathscr{U}$ is a Zariski open subset of $\mathbb{C}^{n}$, all defined over the real field such that:

(i) $h$ is a proper submersive whose components are in involution; that is,

$$
\left\{X_{i} o h, X_{j} o h\right\} \equiv \widetilde{\omega}\left(d\left(X_{i} o h\right), d\left(X_{j} o h\right)\right)=0,
$$

$X_{i}$ being coordinates on $\mathbb{R}^{n}$.

(ii) $M$ is a component of $\mathscr{M}_{\mathbb{R}}$, the $\widetilde{\omega}$ on $M$ is the $\widetilde{\omega}$ on $\mathscr{M}$ along $M$, and $H$ is a $C^{\infty}$-function of $X_{j}$. oh $\left.\right|_{M}$.

2) [6] Let $(M,\{.,\},. \varphi)$ be a complex integrable system where $M$ is a non-singular affine variety and $\varphi=\left(H_{1}, \ldots, H_{s}\right)$ is given by regular algebraic functions $H_{i}$. We say that this system is an a.c.i. system iffor generic $c \in \mathbb{C}^{s}$ the fiber of $\varphi^{-1}(c)$ is an affine part of an Abelian variety and if the Hamiltonian vector fields $X_{H_{i}}$, are translation invariant, when restricted to these fibers.

3) [9] Let $(M,\{.,\}$.$) be a smooth Poisson variety. An a.c.i. Hamiltonian system consists of a proper flat morphism$ $H: M \longrightarrow B$ where $B$ is a smooth variety such that, over the complement $B \backslash \Delta$ of some proper closed subvariety $\Lambda \subset B$, the morphism $H$ is a Lagrangian fibration whose fibers are isomorphic to Abelian varieties. 
4) [18] Let $M$ be a $2 n$-dimensional complex manifold with a holomorphic symplectic structure $\omega$, a holomorphic function $H: M \longrightarrow \mathbb{C}$ and $n$ holomorphic functions which pairwise are in involution and also with $H$, i.e.,

$$
\left\{H_{i}, H_{j}\right\}=\left\{H_{i}, H\right\}=0, \quad 1 \leq i, j \leq n .
$$

Let $B$ be an open dense subset of $\mathbb{C}^{n}$ and

$$
F: F^{-1}(B) \subset M \longrightarrow B
$$

a submersive map. The Hamiltonian system defined by the vector field $X_{H}$ is an a.c.i. system if there exists a bundle $\pi: A \longrightarrow B$ of Abelian varieties, a divisor $D \subset A$, an isomorphism $\sigma: F^{-1}(B) \longrightarrow A \backslash D$, and a vector field $Y$ on $A \backslash D$ which restricts to a linear vector field on the fibers of $\pi$, so that the diagram:

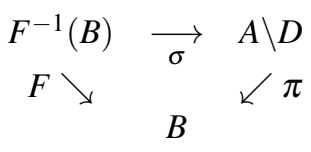

is commutative and such that the vector field $X_{H}$ is $\sigma$-related to $Y$.

5) Hitchin [15] gave a large class of interesting integrable systems that are almost by construction algebraic completely integrable and showed that the cotangent bundle of moduli spaces of stable vector bundles on Riemann surfaces carry the structure of integrable systems and are indeed a.c.i.

It will be interesting to insist a little more on the interpretation of the algebraic complete integrability of a Hamiltonian system, especially in the sense of Adler-van Moerbeke.

Remark 2.4. a) The complete algebraic integrability in the sense of Adler-van Moerbeke in the case where $M=\mathbb{C}^{m}$ means that :

(i) the system (2.1) with polynomial right hand possesses $n+k$ independent polynomial invariants $H_{1} \equiv H_{2} H_{2}, \ldots, H_{n+k}$ of which $k$ invariants lead to zero vector fields, the $n=(m-k) / 2$ remaining ones are in involution, which give rise to $n$ commuting vector fields. For generic $c_{i}$, the invariant manifolds

$$
\bigcap_{i=1}^{n+k}\left\{z \in \mathbb{R}^{m}: H_{i}=c_{i}\right\}
$$

are assumed compact, connected and therefore real tori by the Arnold-Liouville theorem.

(ii) the invariant manifolds, thought of as affine varieties in $\mathbb{C}^{m}$ (non-compact), can be completed into complex algebraic tori, i.e.,

$$
\bigcap_{i=1}^{n+k}\left\{z \in \mathbb{C}^{m}: H_{i}(z)=c_{i}\right\}=T^{n} \backslash\left\{\mathscr{D} \equiv\left(\begin{array}{c}
\text { one or several codimension } \\
\text { one subvarieties }
\end{array}\right)\right\},
$$

where the tori $T^{n}=\mathbb{C}^{n} /$ Lattice = complex algebraic torus (Abelian variety), depend on the c's. In the natural coordinates $\left(t_{1}, \ldots, t_{n}\right)$ of these tori, the Hamiltonian flows (run with complex time) defined by the vector fields generated by the constants of the motion are straight-line motions and the coordinates $z_{i}=z_{i}\left(t_{1}, \ldots, t_{n}\right)$ are meromorphic in $\left(t_{1}, \ldots, t_{n}\right)$.

b) It must be realized that the existence of polynomial first integrals (invariants) for a Hamiltonian system does not necessarily imply the complete integrability of this system. For example, the Hamiltonian system where

$$
H(x, y)=\frac{x^{2}}{2}+P(y), \quad(P(y) \text { being a polynomial in } y),
$$

will be algebraically completely integrable with Abelian (here elliptic) functions if and only if $P(y)$ is a polynomial of degree 3 or 4. Following Mumford [34], the commuting vector fields $X_{H_{1}}, \ldots, X_{H_{n}}$ define on the real torus $M_{c} \subset \mathbb{R}^{2 n}$ defined by the intersection of the constants of the motion $H_{1}=c_{1}, \ldots, H_{n}=c_{n}$, an addition law

$$
\oplus: M_{c} \times M_{c} \longrightarrow M_{c}, \quad(x, y) \longmapsto x \oplus y=g_{t+s}(p), \quad p \in M_{c},
$$

with $x=g_{t}(p), y=g_{s}(p), g_{t}(p)=g_{t_{1}}^{X_{1}} \ldots g_{t_{n}}^{X_{n}}(p)$, where $g_{t_{i}}^{X_{i}}(p)$ denote the flows generated by $X_{H_{i}}$. Algebraic complete integrability means that this addition law is rational, that is:

$$
(x \oplus y)_{j}=R_{j}\left(x_{i}, y_{i}, c\right),
$$


where $R_{j}\left(x_{i}, y_{i}, c\right)$ is a rational function of all coordinates $x_{i}, y_{i}, 1 \leq i \leq n$. Putting $x=p, y=g_{t}^{X_{i}}(p)$, in this formula, we notice that on the real torus $T_{c}$, the flows $g_{t}^{X_{i}}(p)$ depend rationally on the initial condition $p$. Moreover, a Weierstrass theorem on functions admitting an addition theorem states that the coordinates $x_{i}$ are restricted to the real torus:

$$
\mathbb{R}^{n} / \text { Lattice } \longrightarrow M_{c}, \quad\left(t_{1}, \ldots, t_{n}\right) \longmapsto x_{i}\left(t_{1}, \ldots, t_{n}\right)
$$

are Abelian functions. Geometrically, this means that the real torus $M_{c} \simeq \mathbb{R}^{n} /$ Lattice is the affine part of an algebraic complex torus (Abelian variety) $\mathbb{C}^{n} /$ Lattice and that the real functions $x_{i}\left(t_{1}, \ldots, t_{n}\right),\left(t_{i} \in \mathbb{R}\right)$, are the restrictions on this real torus of the meromorphic functions $x_{i}\left(t_{1}, \ldots, t_{n}\right),\left(t_{i} \in \mathbb{C}\right)$, of $n$ complex variables, with $2 n$ periods ( $n$ periods $+n$ imaginary periods). In degenerate situations, some of these periods may be infinite, as for example in the case of a harmonic oscillator.

c) If the Hamiltonian flow (2.1) is a.c.i., it means that the variables $z_{i}$ are meromorphic on the torus $T^{n}$ and by compactness they must blow up along a codimension one subvariety (a divisor) $\mathscr{D} \subset T^{n}$. By the a.c.i. definition, the flow (2.1) is a straight line motion in $T^{n}$ and thus it must hit the divisor $\mathscr{D}$ in at least one place. Moreover through every point of $\mathscr{D}$, there is a straight line motion and therefore a Laurent expansion around that point of intersection. Hence the differential equations must admit Laurent expansions which depend on the $n-1$ parameters defining $\mathscr{D}$ and the $n+k$ constants $c_{i}$ defining the torus $T^{n}$, the total count is therefore $m-1=\operatorname{dim}($ phase space $)-1$ parameters. The fait that a.c.i. systems possess $(m-1)$-dimensional families of Laurent solutions, was implicitly used by Kowalewski [21] in her classification of integrable rigid body motions (see also the Painlevé analysis for ordinary differential equations [35]). Such a necessary condition (see [5]) for a.c.i. can be formulated as in the theorem below.

Theorem 2.5. If the Hamiltonian system (2.1) (with invariant tori not containing elliptic curves) is a.c.i., then each $z_{i}$ blows up after a finite (complex) time, and for every $z_{i}$, there is a family of solutions

$$
z_{i}=\sum_{j=0}^{\infty} z_{i}^{(j)} t^{j-s_{i}}, \quad s_{i} \in \mathbb{Z}, \quad \text { some } s_{i}>0
$$

depending on $\operatorname{dim}($ phase space $)-1=m-1$, free parameters. Moreover, the system (2.1) possesses families of Laurent solutions depending on $m-2, m-3, \ldots, m-n$ free parameters. The coefficients of each one of these solutions are rational functions on affine algebraic varieties of dimensions $m-1, m-2, m-3, \ldots, m-n$.

The question raised several years ago is whether this criterion is also sufficient. The main problem will be to complete the affine variety $M_{c}$ (2.2) into an Abelian variety. A naive guess would be to take the natural compactification $\bar{M}_{c}$ of $M_{c}$ by projectivizing the equations. Indeed, this can never work for a general reason: an Abelian variety $\widetilde{M}_{c}$ of dimension bigger or equal than two is never a complete intersection, that is it can never be described in some projective space $\mathbb{P}^{n}$ by $n$-dim $\widetilde{M}_{c}$ global polynomial homogeneous equations. In other words, if $M_{c}$ is to be the affine part of an Abelian variety, $\bar{M}_{c}$ must have a singularity somewhere along the locus at infinity. The trajectories of the vector fields (2.1) hit every point of the singular locus at infinity and ignore the smooth locus at infinity. In fact, the existence of meromorphic solutions to the differential equations (2.1) depending on some free parameters can be used to manufacture the tori, without ever going through the delicate procedure of blowing up and down. Information about the tori can then be gathered from the divisor. More precisely, around the points of hitting, the system of differential equations (2.1) admit a Laurent expansion solution depending on $m-1$, free parameters and in order to regularize the flow at infinity, we use these parameters to blowing up the variety $\bar{M}_{c}$ along the singular locus at infinity. The new complex variety obtained in this fashion is compact, smooth and has commuting vector fields on it; it is therefore an Abelian variety.

The system (2.1) with $k+n$ polynomial invariants has a coherent tree of Laurent solutions, when it has families of Laurent solutions in $t$, depending on $n-1, n-2, \ldots, m-n$ free parameters. Adler and van Moerbeke [5] have shown that if the system possesses several families of $(n-1)$-dimensional Laurent solutions (principal Painlevé solutions) they must fit together in a coherent way and as we mentioned above, the system must possess $(n-2)-,(n-3)-, \ldots$ dimensional Laurent solutions (lower Painlevé solutions), which are the gluing agents of the $(n-1)$-dimensional family. The gluing occurs via a rational change of coordinates in which the lower parameter solutions are seen to be genuine limits of the higher parameter solutions an which in turn appears due to a remarkable propriety of a.c.i. systems; they can be put into quadratic form both in the original variables and in their ratios (to see further). As a whole, the full set of Painlevé solutions glue together to form a fiber bundle with singular base. A partial converse to the above theorem can be formulated as follows [5]:

Theorem 2.6. If the Hamiltonian system (2.1) satisfies the condition a)(i) in the remark 2.2 of a.c.i. and if it possesses a coherent tree of Laurent solutions, then the system is a.c.i. and there are no other $m-1$-dimensional Laurent solutions but those provided by the coherent set. 
We assume that the divisor is very ample and in addition projectively normal (see [12, 27] for definitions when needed). Consider a point $p \in \mathscr{D}$, a chart $U_{j}$ around $p$ on the torus and a function $y_{j}$ in $\mathscr{L}(\mathscr{D})$ having a pole of maximal order at $p$. Then the vector $\left(1 / y_{j}, y_{1} / y_{j}, \ldots, y_{N} / y_{j}\right)$ provides a good system of coordinates in $U_{j}$. Then taking the derivative with regard to one of the flows

$$
\left(\frac{y_{i}}{y_{j}}\right) \cdot=\frac{\dot{y}_{i} y_{j}-y_{i} \dot{y}_{j}}{y_{j}^{2}}, \quad 1 \leq j \leq N
$$

are finite on $U_{j}$ as well. Therefore, since $y_{j}^{2}$ has a double pole along $\mathscr{D}$, the numerator must also have a double pole (at worst), i.e., $\dot{y}_{i} y_{j}-y_{i} \dot{y}_{j} \in \mathscr{L}(2 \mathscr{D})$. Hence, when $\mathscr{D}$ is projectively normal, we have that

$$
\left(\frac{y_{i}}{y_{j}}\right) \cdot=\sum_{k, l} a_{k, l}\left(\frac{y_{k}}{y_{j}}\right)\left(\frac{y_{l}}{y_{j}}\right),
$$

i.e., the ratios $y_{i} / y_{j}$ form a closed system of coordinates under differentiation. At the bad points, the concept of projective normality play an important role: this enables one to show that $y_{i} / y_{j}$ is a bona fide Taylor series starting from every point in a neighborhood of the point in question. Moreover, the Laurent solutions provide an effective tool for find the constants of the motion. For that, just search polynomials $H_{i}$ of $z$, having the property that evaluated along all the Laurent solutions $z(t)$ they have no polar part. Indeed, since an invariant function of the flow does not blow up along a Laurent solution, the series obtained by substituting the formal solutions (2.3) into the invariants should, in particular, have no polar part. The polynomial functions $H_{i}(z(t))$ being holomorphic and bounded in every direction of a compact space, (i.e., bounded along all principle solutions), are thus constant by a Liouville type of argument. It thus an important ingredient in this argument to use all the generic solutions. To make these informal arguments rigorous is an outstanding question of the subject.

Assume Hamiltonian flows to be weight-homogeneous with a weight $s_{i} \in \mathbb{N}$, going with each variable $z_{i}$, i.e.,

$$
f_{i}\left(\alpha^{s_{1}} z_{1}, \ldots, \alpha^{s_{m}} z_{m}\right)=\alpha^{s_{i}+1} f_{i}\left(z_{1}, \ldots, z_{m}\right), \quad \forall \alpha \in \mathbb{C}
$$

Observe that then the constants of the motion $H$ can be chosen to be weight-homogeneous:

$$
H\left(\alpha^{s_{1}} z_{1}, \ldots, \alpha^{s_{m}} z_{m}\right)=\alpha^{k} H\left(z_{1}, \ldots, z_{m}\right), \quad k \in \mathbb{Z} .
$$

The study of the a.c.i. of Hamiltonian systems, includes several passages to prove rigorously. Here we mention the main passages, leaving the detail when studying the different problems in the following sections. We saw that if the flow is algebraically completely integrable, the differential equations (2.1) must admits Laurent series solutions (2.3) depending on $m-1$ free parameters. We must have $k_{i}=s_{i}$ and coefficients in the series must satisfy at the $0^{t h}$ step non-linear equations,

$$
f_{i}\left(z_{1}^{(0)}, \ldots, z_{m}^{(0)}\right)+g_{i} z_{i}^{(0)}=0,1 \leq i \leq m
$$

and at the $\mathrm{k}^{\text {th }}$ step, linear systems of equations :

$$
(L-k I) z^{(k)}=\left\{\begin{aligned}
0 & \text { for } k=1 \\
\text { some polynomial in } & z^{(1)}, \ldots, z^{(k-1)} \text { for } k>1,
\end{aligned}\right.
$$

where

$$
L=\text { Jacobian map of }(2.4)=\frac{\partial f}{\partial z}+\left.g I\right|_{z=z^{(0)}} .
$$

If $m-1$ free parameters are to appear in the Laurent series, they must either come from the non-linear equations (2.4) or from the eigenvalue problem (2.5), i.e., $L$ must have at least $m-1$ integer eigenvalues. These are much less conditions than expected, because of the fact that the homogeneity $k$ of the constant $H$ must be an eigenvalue of $L$. Moreover the formal series solutions are convergent as a consequence of the majorant method $[6,24]$. Thus, the first step is to show the existence of the Laurent solutions, which requires an argument precisely every time $k$ is an integer eigenvalue of $L$ and therefore $L-k I$ is not invertible. One shows the existence of the remaining constants of the motion in involution so as to reach the number $n+k$. Then you have to prove that for given $c_{1}, \ldots, c_{m}$, the set

$$
\mathscr{D} \equiv\left\{\begin{array}{l}
z_{i}(t)=t^{-v_{i}}\left(z_{i}^{(0)}+z_{i}^{(1)} t+z_{i}^{(2)} t^{2}+\cdots\right), 1 \leq i \leq m \\
\text { Laurent solutions such that }: H_{j}\left(z_{i}(t)\right)=c_{j}+\text { Taylor part }
\end{array}\right\},
$$


defines one or several $n-1$ dimensional algebraic varieties ("Painlevé" divisor) having the property that $M_{c} \cup \mathscr{D}$, is a smooth compact, connected variety with $n$ commuting vector fields independent at every point, i.e., a complex algebraic torus $\mathbb{C}^{n} /$ Lattice. The flows $J \frac{\partial H_{k+i}}{\partial z}, \ldots, J \frac{\partial H_{k+n}}{\partial z}$ are straight line motions on this torus. Let's point out and we'll see all this in more detail later, that having computed the space of functions $\mathscr{L}(\mathscr{D})$ with simple poles at worst along the expansions, it is often important to compute the space of functions $\mathscr{L}(k \mathscr{D})$ of functions having $k$-fold poles at worst along the expansions. These functions play a crucial role in the study of the procedure for embedding the invariant tori into projective space.

The idea of the Adler-van Moerbeke's proof [3] we shall give here is closely related to the geometric spirit of the ArnoldLiouville theorem [7]. Namely, a compact complex $n$-dimensional variety on which there exist $n$ holomorphic commuting vector fields which are independent at every point is analytically isomorphic to a $n$-dimensional complex torus $\mathbb{C}^{n} /$ Lattice and the complex flows generated by the vector fields are straight lines on this complex torus.

Theorem 2.7. Let

$$
\overline{\mathscr{A}}=\bigcap_{i}\left\{Z=\left(Z_{0}, Z_{1}, \ldots, Z_{n}\right) \in \mathbb{P}^{N}(\mathbb{C}): P_{i}(Z)=0\right\},
$$

be an irreducible variety defined by an intersection involving a large number of homogeneous polynomials $P_{i}$ with smooth and irreducible affine part $\mathscr{A}=\overline{\mathscr{A}} \cap\left\{Z_{0} \neq 0\right\}$. Put $\overline{\mathscr{A}} \equiv \mathscr{A} \cup \mathscr{D}$, i.e., $\mathscr{D}=\overline{\mathscr{A}} \cap\left\{Z_{0}=0\right\}$ and consider the map

$$
f: \overline{\mathscr{A}} \longrightarrow \mathbb{P}^{N}(\mathbb{C}), \quad Z \longmapsto f(Z)
$$

Let

$$
\widetilde{\mathscr{A}}=f(\overline{\mathscr{A}})=\overline{f(\mathscr{A})}, \quad \mathscr{D}=\bigcup_{i=1}^{r} \mathscr{D}_{i}, \quad \mathscr{S} \equiv f(\mathscr{D})=\bigcup_{i=1}^{r} f\left(\mathscr{D}_{i}\right) \equiv \bigcup_{i=1}^{r} \mathscr{S}_{i}
$$

where $\mathscr{D}_{i}$ are codimension 1 subvarieties. Assume that:

(i) $f$ maps $\mathscr{A}$ smoothly and 1-1 onto $f(\mathscr{A})$.

(ii) There exist $n$ holomorphic vector fields $X_{1}, \ldots, X_{n}$ on $\mathscr{A}$ which commute and are independent at every point. One vector field, say $X_{k}$ (where $\left.1 \leq k \leq n\right)$, extends holomorphically to a neighborhood of $\mathscr{S}_{k}$ in the projective space $\mathbb{P}^{N}(\mathbb{C})$.

(iii) For all $p \in \mathscr{S}_{k}$, the integral curve $f(t) \in \mathbb{P}^{N}(\mathbb{C})$ of the vector field $X_{k}$ through $f(0)=p \in \mathscr{S}_{k}$ has the property that

$$
\{f(t): 0<|t|<\varepsilon, t \in \mathbb{C}\} \subset f(\mathscr{A}) .
$$

This condition means that the orbits of $X_{k}$ through $\mathscr{S}_{k}$ go immediately into the affine part and in particular, the vector field $X_{k}$ does not vanish on any point of $\mathscr{S}_{k}$.

Then

a) $\widetilde{M}$ is compact, connected and admits an embedding into $\mathbb{P}^{N}(\mathbb{C})$.

b) $\widetilde{\mathscr{A}}$ is diffeomorphic to a $n$-dimensional complex torus. The vector fields $X_{1}, \ldots, X_{n}$ extend holomorphically and remain independent on $\widetilde{\mathscr{A}}$.

c) $\widetilde{\mathscr{A}}$ is a Kähler variety.

d) $\widetilde{M}$ is a Hodge variety. In particular, $\mathscr{A}$ is the affine part of an Abelian variety $\widetilde{\mathscr{A}}$.

Proof. a) A crucial step is to show that the orbits running through $\mathscr{S}_{k}$ form a smooth variety $\Sigma_{p}, p \in \mathscr{S}_{k}$ such that $\Sigma_{p} \backslash \mathscr{S}_{k} \subseteq \mathscr{A}$. Let $p \in \mathscr{S}_{k}, \varepsilon>0$ small enough, $g_{X_{k}}^{t}$ the flow generated by $X_{k}$ on $\mathscr{A}$ and $\left\{g_{X_{k}}^{t}: t \in \mathbb{C}, 0<|t|<\varepsilon\right\}$, the orbit going through the point $p$. The vector field $X_{k}$ is holomorphic in the neighborhood of any point $p \in \mathscr{S}_{k}$ and non-vanishing, by $(i i)$ and $(i i i)$. Then the flow $g_{X_{k}}^{t}$ can be straightened out after a holomorphic change of coordinates. Let $\mathscr{H} \subset \mathbb{P}^{N}(\mathbb{C})$ be a hyperplane transversal to the direction of the flow at $p$ and let $\Sigma_{p}$ be the surface element formed by the divisor $\mathscr{S}_{k}$ and the

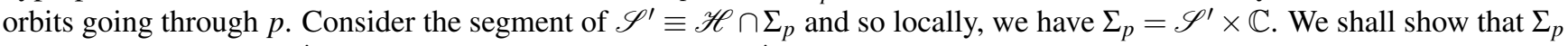
is smooth. Note that $\mathscr{S}^{\prime}$ is smooth. Indeed, suppose that $\mathscr{S}^{\prime}$ is singular at 0 , then $\Sigma_{p}$ would be singular along the trajectory ( $t$-axis) which go immediately into the affine $f(\mathscr{A})$, by condition (iii). Hence, the affine part would be singular which is impossible by condition $(i)$. So, $S^{\prime}$ is smooth and by the implicit function theorem, $\Sigma_{p}$ is smooth too. Consider now the map

$$
\overline{\mathscr{A}} \subset \mathbb{P}^{m}(\mathbb{C}) \longrightarrow \mathbb{P}^{N}(\mathbb{C}), \quad Z \longmapsto f(Z),
$$

where $Z=\left(Z_{0}, Z_{1}, \ldots, Z_{n}\right) \in \mathbb{P}^{m}(\mathbb{C})$ and $\widetilde{\mathscr{A}}=f(\overline{\mathscr{A}})=\overline{f(\mathscr{A})}$. Recall that the flow exists in a full neighborhood of $p$ in $\mathbb{P}^{N}(\mathbb{C})$ and it has been straightened out. Therefore, near $p \in \mathscr{S}_{k}$, we have $\Sigma_{p}=\widetilde{\mathscr{A}}$ and $\Sigma_{p} \backslash \mathscr{S}_{k} \subseteq \mathscr{A}$. Otherwise, there would exist an element $\Sigma_{p}^{\prime} \subset \widetilde{\mathscr{A}}$ such that

$$
\left\{g_{X_{k}}^{t}: t \in \mathbb{C}, 0<|t|<\varepsilon\right\}=\left(\Sigma_{p} \cap \Sigma_{p}^{\prime}\right) \backslash p \subset \mathscr{A}
$$


by condition (iii). In other words, $\Sigma_{p} \cap \Sigma_{p}^{\prime}=t$-axis and hence $\mathscr{A}$ would be singular along the $t$-axis which is impossible. Since the variety $\mathscr{A}$ is irreducible and since the generic hyperplane section $\mathscr{H}_{\text {gen }}$. of $\widetilde{\mathscr{A}}$ is also irreducible, all hyperplane sections are connected and hence $\mathscr{D}$ is also connected. Now consider the graph $G_{f} \subset \mathbb{P}^{m}(\mathbb{C}) \times \mathbb{P}^{N}(\mathbb{C})$ of the map $f$, which is irreducible together with $\widetilde{\mathscr{A}}$. It follows from the irreducibility of $G_{f}$ that a generic hyperplane section $G_{f} \cap\left(\mathscr{H}_{\text {gen. }} \times \mathbb{P}^{N}(\mathbb{C})\right)$ is irreducible, hence the special hyperplane section $G_{f} \cap\left(\left\{Z_{0}=0\right\} \times \mathbb{P}^{N}(\mathbb{C})\right)$ is connected and therefore the projection map

$$
\operatorname{Proj}_{\mathbb{P}^{N}(\mathbb{C})}\left[G_{f} \cap\left(\left\{Z_{0}=0\right\} \times \mathbb{P}^{N}(\mathbb{C})\right)\right]=f(\mathscr{D}) \equiv \mathscr{S}
$$

is connected. Hence, the variety

$$
\widetilde{\mathscr{A}}=\mathscr{A} \cup\left(\cup_{p \in \mathscr{S}_{k}} \Sigma_{p}\right)=\mathscr{A} \cup \mathscr{S}_{k} \subseteq \mathbb{P}^{N}(\mathbb{C}),
$$

is compact, connected and embeds smoothly into $\mathbb{P}^{N}(\mathbb{C})$ via $f$.

$b)$ Let $g^{t_{i}}$ be the flow generated by $X_{i}$ on $\mathscr{A}$ and let $p_{1} \in \widetilde{\mathscr{A}} \backslash \mathscr{A}$. For $\varepsilon>0$ and for all $t_{1} \in \mathbb{C}$ such that $0<\left|t_{1}\right|<\varepsilon$, note that $q \equiv g^{t_{1}}\left(p_{1}\right)$ is well defined and $g^{t_{1}}\left(p_{1}\right) \in f(\mathscr{A})$, using condition (iii). Let $U(q) \subseteq \mathscr{A}$ be a neighborhood of $q$ and let

$$
g^{t_{2}}\left(p_{2}\right)=g^{-t_{1}} \circ g^{t_{2}} \circ g^{t_{1}}\left(p_{2}\right), \quad \forall p_{2} \in U\left(p_{1}\right) \equiv g^{-t_{1}}(U(q)),
$$

which is well defined since by commutativity one can see that the right hand side is independent of $t_{1}$ :

$$
g^{-\left(t_{1}+\varepsilon\right)} \circ g^{t_{2}} \circ g^{t_{1}+\varepsilon}\left(p_{2}\right)=g^{-\left(t_{1}+\varepsilon\right)} \circ g^{t_{2}} \circ g^{t_{1}} \circ g^{\varepsilon}\left(p_{2}\right)=g^{-\left(t_{1}+\varepsilon\right)} \circ g^{\varepsilon} \circ g^{t_{2}} \circ g^{t_{1}}\left(p_{2}\right)=g^{-t_{1}} \circ g^{t_{2}} \circ g^{t_{1}}\left(p_{2}\right) .
$$

Notice that $g^{t_{2}}\left(p_{2}\right)$ is a holomorphic function of $p_{2}$ and $t_{2}$, because in $U\left(p_{1}\right)$ the function $g^{t_{1}}$ is holomorphic and its image is away from $\mathscr{S}$, i.e., in the affine, $g^{t_{2}}$ is holomorphic. The same argument applies to $g^{t_{3}}\left(p_{3}\right), \ldots, g^{t_{n}}\left(p_{n}\right)$ where

$$
g^{t_{n}}\left(p_{n}\right)=g^{-t_{n-1}} \circ g^{t_{n}} \circ g^{t_{n-1}}\left(p_{n}\right), \quad \forall p_{n} \in U\left(p_{n-1}\right) \equiv g^{-t_{n-1}}(U(q)) .
$$

Thus $X_{1}, \ldots, X_{n}$ have been holomorphically extended, remain independent and commuting on $\widetilde{\mathscr{A}}$. Therefore, we can show along the same lines as in the Arnold-Liouville theorem [7] that $\widetilde{\mathscr{A}}$ is a complex torus $\mathbb{C}^{n} /$ lattice. And that will done, by considering the local diffeomorphism

$$
\mathbb{C}^{n} \longrightarrow \widetilde{\mathscr{A}}, \quad t=\left(t_{1}, \ldots, t_{n}\right) \longmapsto g^{t} p=g^{t_{1}} \circ \ldots \circ g^{t_{n}}(p),
$$

for a fixed origin $p \in f(\mathscr{A})$. The additive subgroup $L=\left\{t \in \mathbb{C}^{n}: g^{t} p=p\right\}$, is a lattice of $\mathbb{C}^{n}$ (spanned by $2 n$ vectors in $\mathbb{C}^{n}$, independent over $\mathbb{R}$ ), hence $\mathbb{C}^{n} / L \longrightarrow \widetilde{\mathscr{A}}$ is a biholomorphic diffeomorphism.

c) Let

$$
d s^{2}=\sum_{k=1}^{n} d t_{k} \otimes d \bar{t}_{k}
$$

be a hermitian metric on the complex variety $\widetilde{\mathscr{A}}$ and let $\omega$ its fundamental $(1,1)$-form. We have

$$
\omega=-\frac{1}{2} \operatorname{Im} d s^{2}=\frac{\sqrt{-1}}{2} \sum_{k=1}^{n} d t_{k} \wedge d \bar{t}_{k}
$$

So we see that $\omega$ is closed and the metric $d s^{2}$ is kähler and consequently $\widetilde{\mathscr{A}}$ is a Kähler variety.

d) On the Kähler variety $\widetilde{\mathscr{A}}$ are defined periods of $\omega$. If these periods are integers (possibly after multiplication by a number), we obtain a variety of Hodge. More specifically, integrals $\int_{\gamma_{k}} \omega$ of the form $\omega$ (where $\gamma_{k}$ are cycles in $H_{2}(\widetilde{\mathscr{A}}, \mathbb{Z})$ ) determine the periods $\omega$. As they are integers, then $\widetilde{\mathscr{A}}$ is a Hodge variety. The variety $\widetilde{\mathscr{A}}$ is equipped with $n$ holomorphic vectors fields, independent and commuting. From $a$ ) and $b$ ) the variety $\widetilde{\mathscr{A}}$ is both a projective variety and a complex torus and hence an Abelian variety as a consequence of Chow theorem [12,27]. Another proof is to use the result that we just show since every Hodge torus is Abelian, the converse is also true. Note also that by Moishezon's theorem [30], a compact complex kähler variety having as many independent meromorphic functions as its dimension is an Abelian variety. 


\section{Geodesic flow on the group $S O(3)$ and Euler rigid body motion}

The Euler rigid body motion [11] (also called Euler-Poinsot [36] motion of the solid) can be regarded as geodesic flow on $S O(3)$. This problem expresses the free motion of a rigid body around a fixed point and the motion of the body is governed by the following equation

$$
\dot{M}=[M, \Lambda M],
$$

with

$$
M=\left(M_{i j}\right)_{1 \leq i, j \leq 3} \equiv \sum_{i=1}^{3} m_{i} e_{i} \equiv\left(\begin{array}{ccc}
0 & -m_{3} & m_{2} \\
m_{3} & 0 & -m_{1} \\
-m_{2} & m_{1} & 0
\end{array}\right) \in \operatorname{so}(3),
$$

and

$$
\Lambda M=\sum_{i=1}^{3} \lambda_{i} m_{i} e_{i} \equiv\left(\begin{array}{ccc}
0 & -\lambda_{3} m_{3} & \lambda_{2} m_{2} \\
\lambda_{3} m_{3} & 0 & -\lambda_{1} m_{1} \\
-\lambda_{2} m_{2} & \lambda_{1} m_{1} & 0
\end{array}\right) \in \operatorname{so}(3), \quad \lambda_{i} \equiv \frac{1}{I_{i}},
$$

where $\left(m_{1}, m_{2}, m_{3}\right)$ is the angular momentum, and $I_{1}, I_{2}, I_{3}$, the principal moments of inertia about the principal axes of inertia. Equation (3.1) is written explicitly in the form

$$
\begin{aligned}
& \dot{m}_{1}=\left(\lambda_{3}-\lambda_{2}\right) m_{2} m_{3}, \\
& \dot{m}_{2}=\left(\lambda_{1}-\lambda_{3}\right) m_{1} m_{3}, \\
& \dot{m}_{3}=\left(\lambda_{2}-\lambda_{1}\right) m_{1} m_{2},
\end{aligned}
$$

and can also be written as a Hamiltonian vector field

$$
\dot{x}=J \frac{\partial H}{\partial x}, x=\left(m_{1}, m_{2}, m_{3}\right)^{\top},
$$

with the Hamiltonian

$$
H=\frac{1}{2}\left(\lambda_{1} m_{1}^{2}+\lambda_{2} m_{2}^{2}+\lambda_{3} m_{3}^{2}\right)
$$

and

$$
J=\left(\begin{array}{ccc}
0 & -m_{3} & m_{2} \\
m_{3} & 0 & -m_{1} \\
-m_{2} & m_{1} & 0
\end{array}\right) \in \operatorname{so}(3)
$$

The system (3.2) has beside the energy $H_{1}=H$, a trivial invariant $H_{2}$, i.e., such that : $J \frac{\partial H_{2}}{\partial x}=0$, implying

$$
H_{2}=\frac{1}{2}\left(m_{1}^{2}+m_{2}^{2}+m_{3}^{2}\right) .
$$

The system evolves on the intersection of the sphere $H_{1}=c_{1}$ and the ellipsoid $H_{2}=c_{2}$. In $\mathbb{R}^{3}$, this intersection will be isomorphic to two circles $\left(\right.$ with $\left.\frac{c_{2}}{\lambda_{3}}<c_{1}<\frac{c_{2}}{\lambda_{1}}\right)$. The system (3.2) is completely integrable and the vector $J \frac{\partial H}{\partial x}$ gives a flow on a variety

$$
M_{c}=\bigcap_{i=1}^{2}\left\{x \in \mathbb{R}^{3}: H_{i}(x)=c_{i}\right\}, \quad\left(c=\left(c_{1}, c_{2}\right) \text { is not a critical value }\right),
$$

diffeomorphic to a real torus of dimension 1 , that is to say a circle.

From the first integrals $H_{1}$ and $H_{2}$, we express $m_{1}$ and $m_{3}$ as a function of $m_{2}$. These expressions are then introduced into the second equation of the system (3.2) to obtain a differential equation in $m_{2}$ and $\frac{d m_{2}}{d t}$ only. In more detail, the following relationships are easily obtained from equations $H_{1}=c_{1}$ and $H_{2}=c_{2}$ :

$$
m_{1}^{2}=\frac{2 c_{1}-r^{2} \lambda_{3}-\left(\lambda_{2}-\lambda_{3}\right) m_{2}^{2}}{\lambda_{1}-\lambda_{3}}
$$




$$
m_{3}^{2}=\frac{2 c_{2} \lambda_{1}-2 c_{1}-\left(\lambda_{1}-\lambda_{2}\right) m_{2}^{2}}{\lambda_{1}-\lambda_{3}}
$$

By substituting these expressions in the second equation of the system (3.2), we obtain

$$
\dot{m}_{2}=\sqrt{\left(2 c_{1}-2 c_{2} \lambda_{3}-\left(\lambda_{2}-\lambda_{3}\right) m_{2}^{2}\right)\left(2 c_{2} \lambda_{1}-2 c_{1}-\left(\lambda_{1}-\lambda_{2}\right) m_{2}^{2}\right)} .
$$

By integrating this equation, we obtain a function $t\left(m_{2}\right)$ in the form of an elliptic integral. To reduce this to the standard form, we can assume that $2 c_{2}>\frac{2 c_{1}}{\lambda_{2}}$ (otherwise, it is enough to invert the indices 1 and 3 in all the previous formulas). We rewrite the previous equation, in the form

$$
\frac{d m_{2}}{\sqrt{\left(2 c_{1}-2 c_{2} \lambda_{3}\right)\left(2 c_{2} \lambda_{1}-2 c_{1}\right)} d t}=\sqrt{\left(1-\frac{\left(\lambda_{2}-\lambda_{3}\right) m_{2}^{2}}{2 c_{1}-2 c_{2} \lambda_{3}}\right)\left(1-\frac{\left(\lambda_{1}-\lambda_{2}\right) m_{2}^{2}}{2 c_{2} \lambda_{1}-2 c_{1}}\right)} .
$$

By setting

$$
\tau=t \sqrt{\left(\lambda_{2}-\lambda_{3}\right)\left(2 c_{2} \lambda_{1}-2 c_{1}\right)}, \quad s=m_{2} \sqrt{\frac{\lambda_{2}-\lambda_{3}}{2 c_{1}-2 c_{2} \lambda_{3}}},
$$

we obtain

$$
\frac{d s}{d \tau}=\sqrt{\left(1-s^{2}\right)\left(1-\frac{\left(\lambda_{1}-\lambda_{2}\right)\left(2 c_{1}-2 c_{2} \lambda_{3}\right)}{\left(\lambda_{2}-\lambda_{3}\right)\left(2 c_{2} \lambda_{1}-2 c_{1}\right)} s^{2}\right)}
$$

which suggests choosing elliptic functions as a module

$$
k^{2}=\frac{\left(\lambda_{1}-\lambda_{2}\right)\left(2 c_{1}-2 c_{2} \lambda_{3}\right)}{\left(\lambda_{2}-\lambda_{3}\right)\left(2 c_{2} \lambda_{1}-2 c_{1}\right)} .
$$

Inequalities $\lambda_{1}>\lambda_{2}>\lambda_{3}, \frac{2 c_{1}}{\lambda_{1}}<2 c_{2}<\frac{2 c_{1}}{\lambda_{3}}$ and $r^{2}>\frac{2 c_{1}}{\lambda_{2}}$ show that $0<k^{2}<1$. So we get

$$
\frac{d s}{d \tau}=\sqrt{\left(1-s^{2}\right)\left(1-k^{2} s^{2}\right)}
$$

This equation admits the solution (we choose the origin of the times such that $m_{2}=0$ for $t=0$ ):

$$
\tau=\int_{0}^{s} \frac{d s}{\sqrt{\left(1-s^{2}\right)\left(1-k^{2} s^{2}\right)}} .
$$

It is the integral of a holomorphic differential on an elliptic curve

$$
\mathscr{E}: w^{2}=\left(1-s^{2}\right)\left(1-k^{2} s^{2}\right) .
$$

The inverse function $s(\tau)$ is one of Jacobi's elliptic functions [27] $: s=\mathbf{s n} \tau$, which also determines $m_{2}$ as a function of time, that is,

$$
m_{2}=\sqrt{\frac{2 H_{1}-r^{2} \lambda_{3}}{\lambda_{2}-\lambda_{3}}} \cdot \mathbf{s n} \tau
$$

According to the equalities (3.3) and (3.4), we know that the functions $m_{1}$ and $m_{3}$ are expressed algebraically as a function of $m_{2}$, so

$$
\begin{aligned}
& m_{1}=\sqrt{\frac{2 H_{1}-r^{2} \lambda_{3}}{\lambda_{1}-\lambda_{3}}} \cdot \sqrt{1-\mathbf{s n}^{2} \tau} \\
& m_{3}=\sqrt{\frac{r^{2} \lambda_{1}-2 H_{1}}{\lambda_{1}-\lambda_{3}}} \cdot \sqrt{1-k^{2} \mathbf{s n}^{2} \tau} .
\end{aligned}
$$


Given the definition of the other two elliptical functions [27] :

$$
\operatorname{cn} \tau=\sqrt{1-\mathbf{s n}^{2} \tau}, \quad \operatorname{dn} \tau=\sqrt{1-k^{2} \mathbf{s n}^{2} \tau}
$$

and the fact that $\tau=t \sqrt{\left(\lambda_{2}-\lambda_{3}\right)\left(2 c_{2} \lambda_{1}-2 c_{1}\right)}$, we finally get the following explicit formulas :

$$
\begin{aligned}
& m_{1}=\sqrt{\frac{2 c_{1}-2 c_{2} \lambda_{3}}{\lambda_{1}-\lambda_{3}}} \mathbf{c n}\left(t \sqrt{\left(\lambda_{2}-\lambda_{3}\right)\left(2 c_{2} \lambda_{1}-2 H_{1}\right)}\right) \\
& m_{2}=\sqrt{\frac{2 c_{1}-2 c_{2} \lambda_{3}}{\lambda_{2}-\lambda_{3}}} \mathbf{s n}\left(t \sqrt{\left(\lambda_{2}-\lambda_{3}\right)\left(2 c_{2} \lambda_{1}-2 c_{1}\right)}\right) \\
& m_{3}=\sqrt{\frac{2 c_{2} \lambda_{1}-2 c_{1}}{\lambda_{1}-\lambda_{3}}} \mathbf{d n}\left(t \sqrt{\left(\lambda_{2}-\lambda_{3}\right)\left(2 c_{2} \lambda_{1}-2 c_{1}\right)}\right) .
\end{aligned}
$$

In other words, the integration of the Euler equations is done by means of elliptic Jacobi functions. In fact, the Euler rigid body motion is always algebraically completely integrable. The two circles of the intersection $M_{c}$, (with $\frac{c_{1}}{\lambda_{3}}<c_{2}<\frac{c_{1}}{\lambda_{1}}$, otherwise it is empty) forms the real part of a complex torus of dimension 1, defined by the elliptic curve $\mathscr{E}(3.5)$. The complex intersection $\left(\subset \mathbb{C}^{3}\right)$ is the affine part of an elliptic curve

$$
\bar{M}_{c}=\left\{X \in \mathbb{P}^{3}(\mathbb{C}): H_{1}(X)=c_{1} X_{0}^{2}\right\} \cap\left\{X \in \mathbb{P}^{3}(\mathbb{C}): H_{2}(X)=c_{2} X_{0}^{2}\right\} .
$$

We show that $\bar{M}_{c}$ is isomorphic to the elliptic curve $\mathscr{E}$. In addition, the circle defined by $\left\{H_{1}=c_{1}\right\} \cap\left\{H_{2}=c_{2}\right\}$ extends to the complex torus $\mathbb{C} /$ lattice and the flow linearizes on this torus. If $p(t)=\left(m_{1}(t), m_{2}(t), m_{3}(t)\right)$, is a solution of the system (3.2), the law connecting $p\left(t_{1}+t_{2}\right)$ to $p\left(t_{1}\right)$ and $p\left(t_{2}\right)$ is the the addition law on the elliptic curve $\mathscr{E}$. From equations $(3.2)$, the unique holomorphic differential on $\bar{M}_{c}$ is given by

$$
\omega=\frac{d m_{1}}{\left(\lambda_{3}-\lambda_{2}\right) m_{2} m_{3}}=\frac{d m_{2}}{\left(\lambda_{1}-\lambda_{3}\right) m_{1} m_{3}}=\frac{d m_{3}}{\left(\lambda_{2}-\lambda_{1}\right) m_{1} m_{2}}
$$

so $t=\int_{p(0)}^{p(t)} \omega, p(0) \in \bar{M}_{c}$. The system (3.2) is invariant by the transformations $t \rightarrow \alpha^{-1} t, m_{1} \rightarrow \alpha m_{1}, m_{2} \rightarrow \alpha m_{2}, m_{3} \rightarrow \alpha m_{3}$. We seek solutions of the system (3.2) or of equation (3.1) in the form of Laurent series

$$
M(t)=t^{-1}\left(M^{(0)}+M^{(1)} t+M^{(2)} t^{2}+\cdots\right)=\sum_{j=0}^{\infty} M^{(j)} t^{j-1}
$$

depending on $\operatorname{dim}($ phase space $)-1=2$ free parameters. Substituting (3.6) into equation (3.1), one obtains

$$
\sum_{j=0}^{\infty}(j-1) M^{(j)} t^{j-2}=\sum_{j=0}^{\infty}\left(\sum_{i=0}^{j}\left[M^{(i)}, \Lambda M^{(j-i)}\right]\right) t^{j-2} .
$$

Therefore,

$$
(j-i) M^{(j)}=\sum_{i=0}^{j}\left[M^{(i)}, \Lambda M^{(j-i)}\right],
$$

and we see that the coefficients $M^{(0)}, M^{(1)}, \ldots$, satisfy the equations

$$
\begin{aligned}
& M^{(0)}+\left[M^{(0)}, \Lambda M^{(0)}\right]=0, \\
& (L-k I) M^{(k)}=-\sum_{i=1}^{k-1}\left[M^{(i)}, \Lambda C^{(k-i)}\right] \quad k \geq 1,
\end{aligned}
$$

where $L$ Is the linear operator $L: s o(3) \longrightarrow s o(3)$ defined by

$$
L(Y)=Y+\left[M^{(0)}, \Lambda Y\right]+\left[Y, \Lambda M^{0}\right]=\text { Jacobian of (3.7). }
$$


The matrix $M^{(0)}$ appearing in $L$ is a solution of the nonlinear equation (3.7). A simple calculation shows that the matrix $(L-k I)$ is always invertible except for $k=2$ and therefore its rank is equal to 1 . This shows that the coefficient $M^{(2)}$ contains two free parameters and can be assimilated to $c_{1}$ and $c_{2}$. In a detailed and explicit way, let us look for the solutions of the system (3.2) in the form of Laurent's series

$$
\begin{aligned}
& m_{1}=\frac{1}{t}\left(m_{1}^{(0)}+m_{1}^{(1)} t+m_{1}^{(2)} t^{2}+\cdots\right), \\
& m_{2}=\frac{1}{t}\left(m_{2}^{(0)}+m_{2}^{(1)} t+m_{2}^{(2)} t^{2}+\cdots\right), \\
& m_{3}=\frac{1}{t}\left(m_{3}^{(0)}+m_{3}^{(1)} t+m_{3}^{(2)} t^{2}+\cdots\right),
\end{aligned}
$$

depending on $\operatorname{dim}$ (phase space) $-1=2$ free parameters. Substituting these equations into the system (3.2), we see that

1) the coefficients $m_{1}^{(0)}, m_{2}^{(0)}, m_{3}^{(0)}$, satisfy the equations

$$
\begin{aligned}
& m_{1}^{(0)}+\left(\lambda_{3}-\lambda_{2}\right) m_{2}^{(0)} m_{3}^{(0)}=0, \\
& m_{2}^{(0)}+\left(\lambda_{1}-\lambda_{3}\right) m_{1}^{(0)} m_{3}^{(0)}=0, \\
& m_{3}^{(0)}+\left(\lambda_{2}-\lambda_{1}\right) m_{1}^{(0)} m_{2}^{(0)}=0,
\end{aligned}
$$

whose solutions are

$$
\begin{aligned}
& 1^{\text {st }} \text { case : } \\
& m_{1}^{(0)}=\frac{-1}{\sqrt{\left(\lambda_{2}-\lambda_{1}\right)\left(\lambda_{1}-\lambda_{3}\right)}}, \quad m_{2}^{(0)}=\frac{1}{\sqrt{\left(\lambda_{2}-\lambda_{1}\right)\left(\lambda_{3}-\lambda_{2}\right)}}, \quad m_{3}^{(0)}=\frac{1}{\sqrt{\left(\lambda_{1}-\lambda_{3}\right)\left(\lambda_{3}-\lambda_{2}\right)}} . \\
& 2^{\text {nd }} \text { case : } \\
& m_{1}^{(0)}=\frac{1}{\sqrt{\left(\lambda_{2}-\lambda_{1}\right)\left(\lambda_{1}-\lambda_{3}\right)}}, \quad m_{2}^{(0)}=\frac{1}{\sqrt{\left(\lambda_{2}-\lambda_{1}\right)\left(\lambda_{3}-\lambda_{2}\right)}}, \quad m_{3}^{(0)}=\frac{-1}{\sqrt{\left(\lambda_{1}-\lambda_{3}\right)\left(\lambda_{3}-\lambda_{2}\right)}} . \\
& 3^{\text {rd }} \text { case : } \\
& m_{1}^{(0)}=\frac{1}{\sqrt{\left(\lambda_{2}-\lambda_{1}\right)\left(\lambda_{1}-\lambda_{3}\right)}}, \quad m_{2}^{(0)}=\frac{-1}{\sqrt{\left(\lambda_{2}-\lambda_{1}\right)\left(\lambda_{3}-\lambda_{2}\right)}}, \quad m_{3}^{(0)}=\frac{1}{\sqrt{\left(\lambda_{1}-\lambda_{3}\right)\left(\lambda_{3}-\lambda_{2}\right)}} . \\
& 4^{\text {th }} \text { case : } \\
& m_{1}^{(0)}=\frac{-1}{\sqrt{\left(\lambda_{2}-\lambda_{1}\right)\left(\lambda_{1}-\lambda_{3}\right)}}, \quad m_{2}^{(0)}=\frac{-1}{\sqrt{\left(\lambda_{2}-\lambda_{1}\right)\left(\lambda_{3}-\lambda_{2}\right)}}, \quad m_{3}^{(0)}=\frac{-1}{\sqrt{\left(\lambda_{1}-\lambda_{3}\right)\left(\lambda_{3}-\lambda_{2}\right)}} .
\end{aligned}
$$

2) the coefficients $m_{1}^{(1)}, m_{2}^{(1)}, m_{3}^{(1)}$, satisfy equations

$$
\begin{aligned}
& \left(\lambda_{3}-\lambda_{2}\right) m_{2}^{(0)} m_{3}^{(1)}+\left(\lambda_{3}-\lambda_{2}\right) m_{2}^{(1)} m_{3}^{(0)}=0, \\
& \left(\lambda_{1}-\lambda_{3}\right) m_{1}^{(0)} m_{3}^{(1)}+\left(\lambda_{1}-\lambda_{3}\right) m_{1}^{(1)} m_{3}^{(0)}=0, \\
& \left(\lambda_{2}-\lambda_{1}\right) m_{1}^{(0)} m_{2}^{(1)}+\left(\lambda_{2}-\lambda_{1}\right) m_{1}^{(1)} m_{2}^{(0)}=0,
\end{aligned}
$$

the solutions of which are in all cases : $m_{1}^{(1)}=m_{2}^{(1)}=m_{3}^{(1)}=0$.

3 ) the coefficients $m_{1}^{(2)}, m_{2}^{(2)}, m_{3}^{(2)}$, satisfy equations

$$
\begin{aligned}
m_{1}^{(2)}-\lambda_{3} m_{2}^{(0)} m_{3}^{(2)}- & \lambda_{3} m_{2}^{(1)} m_{3}^{(1)}-\lambda_{3} m_{2}^{(2)} m_{3}^{(0)} \\
& +\lambda_{2} m_{2}^{(0)} m_{3}^{(2)}+\lambda_{2} m_{2}^{(1)} m_{3}^{(1)}+\lambda_{2} m_{2}^{(2)} m_{3}^{(0)}=0 \\
m_{2}^{(2)}-\lambda_{1} m_{1}^{(0)} m_{3}^{(2)}- & \lambda_{1} m_{1}^{(1)} m_{3}^{(1)}-\lambda_{1} a_{2} m_{3}^{(0)} \\
& +\lambda_{3} m_{1}^{(0)} m_{3}^{(2)}+\lambda_{3} m_{1}^{(1)} m_{3}^{(1)}+\lambda_{3} m_{1}^{(2)} m_{3}^{(0)}=0 \\
m_{3}^{(2)}-\lambda_{2} m_{1}^{(0)} b_{2}-\lambda_{2} m_{1}^{(1)} m_{2}^{(1)}-\lambda_{2} m_{1}^{(2)} m_{2}^{(0)} & +\lambda_{1} m_{1}^{(0)} m_{2}^{(2)}+\lambda_{1} m_{1}^{(1)} m_{2}^{(1)}+\lambda_{1} m_{1}^{(2)} m_{2}^{(0)}=0
\end{aligned}
$$

whose solutions corresponding to the different cases are respectively,

$$
\begin{aligned}
& 1^{\text {st }} \text { case }: m_{1}^{(2)}=\frac{\sqrt{\left(\lambda_{3}-\lambda_{2}\right)}}{\sqrt{\left(\lambda_{1}-\lambda_{3}\right)}} m_{2}^{(2)}+\frac{\sqrt{\left(\lambda_{3}-\lambda_{2}\right)}}{\sqrt{\left(\lambda_{2}-\lambda_{1}\right)}} m_{3}^{(2)} . \\
& 2^{\text {nd }} \text { case }: m_{1}^{(2)}=-\frac{\sqrt{\lambda_{3}-\lambda_{2}}}{\sqrt{\lambda_{1}-\lambda_{3}}} m_{2}^{(2)}+\frac{\sqrt{\lambda_{3}-\lambda_{2}}}{\sqrt{\lambda_{2}-\lambda_{1}}} m_{3}^{(2)} . \\
& 3^{r d} \text { case }: m_{1}^{(2)}=\frac{\sqrt{\lambda_{3}-\lambda_{2}}}{\sqrt{\lambda_{1}-\lambda_{3}}} m_{2}^{(2)}-\frac{\sqrt{\lambda_{3}-\lambda_{2}}}{\sqrt{\lambda_{2}-\lambda_{1}}} m_{3}^{(2)} .
\end{aligned}
$$




$$
4^{\text {th }} \text { case }: m_{1}^{(2)}=-\frac{\sqrt{\lambda_{3}-\lambda_{2}}}{\sqrt{\lambda_{1}-\lambda_{3}}} m_{2}^{(2)}-\frac{\sqrt{\lambda_{3}-\lambda_{2}}}{\sqrt{\lambda_{2}-\lambda_{1}}} m_{3}^{(2)}
$$

where $m_{2}^{(2)}$ et $m_{3}^{(2)}$ are two free parameters.

Therefore, for the first case, we have

$$
\begin{aligned}
& m_{1}=\frac{-1}{t \sqrt{\left(\lambda_{2}-\lambda_{1}\right)\left(\lambda_{1}-\lambda_{3}\right)}}+\left(\frac{\sqrt{\left(\lambda_{3}-\lambda_{2}\right)}}{\sqrt{\left(\lambda_{1}-\lambda_{3}\right)}} m_{2}^{(2)}+\frac{\sqrt{\left(\lambda_{3}-\lambda_{2}\right)}}{\sqrt{\left(\lambda_{2}-\lambda_{1}\right)}} m_{3}^{(2)}\right) t+\cdots, \\
& m_{2}=\frac{1}{t \sqrt{\left(\lambda_{2}-\lambda_{1}\right)\left(\lambda_{3}-\lambda_{2}\right)}}+m_{2}^{(2)} t+\cdots, \\
& m_{3}=\frac{1}{t \sqrt{\left(\lambda_{1}-\lambda_{3}\right)\left(\lambda_{3}-\lambda_{2}\right)}}+m_{3}^{(2)} t+\cdots .
\end{aligned}
$$

Substituting these developments into the first integrals $H_{1}$ and $H_{2}$, we get

$$
\begin{aligned}
& H_{1}=2 \frac{\sqrt{\lambda_{3}-\lambda_{2}}}{\sqrt{\lambda_{2}-\lambda_{1}}}\left(\frac{1}{\lambda_{3}-\lambda_{2}}-\frac{1}{\lambda_{1}-\lambda_{3}}\right) m_{2}^{(2)}+2 \frac{\sqrt{\lambda_{3}-\lambda_{2}}}{\sqrt{\lambda_{1}-\lambda_{3}}}\left(\frac{1}{\lambda_{3}-\lambda_{2}}-\frac{1}{\lambda_{2}-\lambda_{1}}\right) m_{3}^{(2)}, \\
& H_{2}=2 \frac{\sqrt{\lambda_{3}-\lambda_{2}}}{\sqrt{\lambda_{2}-\lambda_{1}}}\left(\frac{\lambda_{2}}{\lambda_{3}-\lambda_{2}}-\frac{\lambda_{1}}{\lambda_{1}-\lambda_{3}}\right) m_{2}^{(2)}+2 \frac{\sqrt{\lambda_{3}-\lambda_{2}}}{\sqrt{\lambda_{1}-\lambda_{3}}}\left(\frac{\lambda_{3}}{\lambda_{3}-\lambda_{2}}-\frac{\lambda_{1}}{\lambda_{2}-\lambda_{1}}\right) m_{3}^{(2)},
\end{aligned}
$$

and we deduce the relations :

$$
\begin{aligned}
& m_{3}^{(2)}=\frac{1}{6 \sqrt{\left(\lambda_{1}-\lambda_{3}\right)\left(\lambda_{3}-\lambda_{2}\right)}}\left(\left(\lambda_{3}-\lambda_{2}\right)\left(\lambda_{1} H_{1}-H_{2}\right)-\left(\lambda_{1}-\lambda_{3}\right)\left(\lambda_{2} H_{1}-H_{2}\right)\right), \\
& m_{2}^{(2)}=\frac{1}{6 \sqrt{\left(\lambda_{2}-\lambda_{1}\right)\left(\lambda_{3}-\lambda_{2}\right)}}\left(\left(\lambda_{2}-\lambda_{1}\right)\left(\lambda_{3} H_{1}-H_{2}\right)-\left(\lambda_{3}-\lambda_{2}\right)\left(\lambda_{1} H_{1}-H_{2}\right)\right) .
\end{aligned}
$$

Obviously, similar expressions are obtained for the other cases.

We deduce from what precedes the following result:

Theorem 3.1. The Euler rigid body motion is always algebraically completely integrable and can be regarded as geodesic flow on $\mathrm{SO}(3)$. In addition, the integration of the equations is done by means of elliptic Jacobi functions.

\section{Clebsch and Lyapunov-Steklov cases of a solid in an ideal fluid}

The equations of motion of a solid in an ideal fluid have the form (Kirchhoff's equations [17]) :

$$
\begin{aligned}
\dot{p}_{1} & =p_{2} \frac{\partial H}{\partial l_{3}}-p_{3} \frac{\partial H}{\partial l_{2}}, \\
\dot{p}_{2} & =p_{3} \frac{\partial H}{\partial l_{1}}-p_{1} \frac{\partial H}{\partial l_{3}}, \\
\dot{p}_{3} & =p_{1} \frac{\partial H}{\partial l_{2}}-p_{2} \frac{\partial H}{\partial l_{1}}, \\
\dot{l}_{1} & =p_{2} \frac{\partial H}{\partial p_{3}}-p_{3} \frac{\partial H}{\partial p_{2}}+l_{2} \frac{\partial H}{\partial l_{3}}-l_{3} \frac{\partial H}{\partial l_{2}} \\
\dot{l}_{2} & =p_{3} \frac{\partial H}{\partial p_{1}}-p_{1} \frac{\partial H}{\partial p_{3}}+l_{3} \frac{\partial H}{\partial l_{1}}-l_{1} \frac{\partial H}{\partial l_{3}} \\
\dot{l}_{3} & =p_{1} \frac{\partial H}{\partial p_{2}}-p_{2} \frac{\partial H}{\partial p_{1}}+l_{1} \frac{\partial H}{\partial l_{2}}-l_{2} \frac{\partial H}{\partial l_{1}},
\end{aligned}
$$

where $\left(p_{1}, p_{2}, p_{3}\right)$ is the velocity of a point fixed relatively to the solid, $\left(l_{1}, l_{2}, l_{3}\right)$ the angular velocity of the body expressed with regard to a frame of reference also fixed relatively to the solid and $H$ is the Hamiltonian. Equations (4.1) can be regarded as the equations of the geodesics of the right-invariant metric on the group $E(3)=S O(3) \times \mathbb{R}^{3}$ of motions of 3-dimensional Euclidean space $\mathbb{R}^{3}$, generated by rotations and translations.

Equations (4.1) have the trivial first integrals (or invariants):

$$
H_{1}=H, \quad H_{2}=\sum_{k=1}^{3} p_{k}^{2}, \quad H_{3}=\sum_{k=1}^{3} p_{k} l_{k} .
$$

We distinguish two integrable cases: the case of Clebsch [8] and the case of Lyapunov-Steklov [28, 37]. 


\subsection{Clebsch's case}

In Clebsch's case, we have

$$
H_{1}=H=\frac{1}{2} \sum_{k=1}^{3}\left(a_{k} p_{k}^{2}+b_{k} l_{k}^{2}\right),
$$

with

$$
\frac{a_{2}-a_{3}}{b_{1}}+\frac{a_{3}-a_{1}}{b_{2}}+\frac{a_{1}-a_{2}}{b_{3}}=0
$$

An additional integral is

$$
H_{4}=\frac{1}{2} \sum_{k=1}^{3}\left(b_{k} p_{k}^{2}+\rho l_{k}^{2}\right),
$$

where the constant $\rho$ satisfies the conditions

$$
\rho=\frac{b_{1}\left(b_{2}-b_{3}\right)}{a_{2}-a_{3}}=\frac{b_{2}\left(b_{3}-b_{1}\right)}{a_{3}-a_{1}}=\frac{b_{3}\left(b_{1}-b_{2}\right)}{a_{1}-a_{2}} .
$$

We shall study briefly Kötter's solution [19] by quadratures of the equations (4.1), in terms of genus 2 hyperelliptic integrals. In fact, the transformation to the separating coordinates $s_{1}$ and $s_{2}$ which leads to the quadratures in terms of hyperelliptic integrals is quite involved. Finding this transformation require a great deal of luck and ingenuity. After the substitution $b_{k} \rightarrow \rho b_{k}$, $1 \leq k \leq 3$, and after an appropriate linear combination of $H_{1}$ and $H_{2}$, the equations (4.2), (4.3), (4.4) can be written in the form

$$
\begin{aligned}
& p_{1}^{2}+p_{2}^{2}+p_{3}^{2}=A, \\
& b_{1} p_{1}^{2}+b_{2} p_{2}^{2}+b_{3} p_{3}^{2}+l_{1}^{2}+l_{2}^{2}+l_{3}^{2}=B, \\
& b_{1} l_{1}^{2}+b_{2} l_{2}^{2}+b_{3} l_{3}^{2}-b_{2} b_{3} p_{1}^{2}-b_{1} b_{3} p_{2}^{2}-b_{1} b_{2} p_{3}^{2}=C, \\
& p_{1} l_{1}+p_{2} l_{2}+p_{3} l_{3}=D,
\end{aligned}
$$

where $A, B, C, D$ are constants. Following [19,10,23], we introduce coordinates $\varphi_{k}, \psi_{k}, 1 \leq k \leq 3$ by setting

$$
\begin{aligned}
& \varphi_{k}=p_{k}\left(\frac{\sqrt{\Pi_{j=1}^{3}\left(z_{1}-b_{j}\right)}}{\sqrt{z_{1}-b_{k}} \sqrt{\partial R / \partial z_{1}}}+\sqrt{-1} \frac{\sqrt{\Pi_{j=1}^{3}\left(z_{2}-b_{j}\right)}}{\sqrt{z_{2}-b_{k}} \sqrt{\partial R / \partial z_{2}}}\right)+l_{k}\left(\frac{\sqrt{z_{1}-b_{k}}}{\sqrt{\partial R / \partial z_{1}}}+\sqrt{-1} \frac{\sqrt{z_{2}-b_{k}}}{\sqrt{\partial R / \partial z_{2}}}\right), \\
& \psi_{k}=p_{k}\left(\frac{\sqrt{\Pi_{j=1}^{3}\left(z_{1}-b_{j}\right)}}{\sqrt{z_{1}-b_{k}} \sqrt{\partial R / \partial z_{1}}}-\sqrt{-1} \frac{\sqrt{\prod_{j=1}^{3}\left(z_{2}-b_{j}\right)}}{\sqrt{z_{2}-b_{k}} \sqrt{\partial R / \partial z_{2}}}\right)+l_{k}\left(\frac{\sqrt{z_{1}-b_{k}}}{\sqrt{\partial R / \partial z_{1}}}-\sqrt{-1} \frac{\sqrt{z_{2}-b_{k}}}{\sqrt{\partial R / \partial z_{2}}}\right),
\end{aligned}
$$

where

$$
R(z)=\prod_{i=1}^{4}\left(z-z_{i}\right),
$$

and $z_{1}, z_{2}, z_{3}, z_{4}$ are the roots of the equation

$$
A^{2}\left(z^{2}-z \sum_{k=1}^{3} b_{k}\right)+B z-C+2 D \sqrt{\prod_{k=1}^{3}\left(z-b_{k}\right)}=0 .
$$

Let $s_{1}$ and $s_{2}$ be the roots of the equation

$$
\frac{\psi_{1}^{2}}{v_{1}^{2}-s}+\frac{\psi_{2}^{2}}{v_{2}^{2}-s}+\frac{\psi_{3}^{2}}{v_{3}^{2}-s}=0
$$

where

$$
v_{k}=\frac{\frac{\sqrt{z_{3}-b_{k}}}{\sqrt{\partial R / \partial z_{3}}}+\sqrt{-1} \frac{\sqrt{z_{4}-b_{k}}}{\sqrt{\partial R / \partial z_{4}}}}{\frac{\sqrt{z_{1}-b_{k}}}{\sqrt{\partial R / \partial z_{1}}}+\sqrt{-1} \frac{\sqrt{z_{2}-b_{k}}}{\sqrt{\partial R / \partial z_{2}}}}, \quad 1 \leq k \leq 3 .
$$


An expression of the original variables $p_{1}, p_{2}, p_{3}, l_{1}, l_{2}, l_{3}$ in terms of $s_{1}$ and $s_{2}$ can be found in [19]. After some algebraic manipulations, we obtain the following equations for $s_{1}$ and $s_{2}$ :

$$
\begin{aligned}
\frac{d s_{1}}{d t} & =\frac{\left(a s_{1}+b\right) \sqrt{P_{5}\left(s_{1}\right)}}{s_{2}-s_{1}} \\
\frac{d s_{2}}{d t} & =\frac{\left(a s_{2}+b\right) \sqrt{P_{5}\left(s_{2}\right)}}{s_{1}-s_{2}}
\end{aligned}
$$

where $a, b$ are constants and $P_{5}(s)$ is a polynomial of degree 5 of the form

$$
P_{5}(s)=s\left(s-v_{1}^{2}\right)\left(s-v_{2}^{2}\right)\left(s-v_{3}^{2}\right)\left(s-v_{1}^{2} v_{2}^{2} v_{3}^{2}\right) .
$$

These equations can be integrated by the Abelian mapping

$$
\mathscr{H} \rightarrow \operatorname{Jac}(\mathscr{H})=\mathbb{C}^{2} / \Lambda, \quad P \longmapsto\left(\int_{P_{0}}^{P} \theta_{1}, \int_{P_{0}}^{P} \theta_{2}\right),
$$

where the hyperelliptic curve $\mathscr{H}$ of genus 2 is given by the equation $w^{2}=P_{5}(s), \Lambda$ is the lattice generated by the vectors $n_{1}+M n_{2},\left(n_{1}, n_{2}\right) \in \mathbb{Z}^{2}, M$ is the matrix of period of the curve $\mathscr{H},\left(\theta_{1}, \theta_{2}\right)$ is a canonical basis of holomorphic differentials on $\mathscr{H}$, i.e.,

$$
\theta_{1}=\frac{d s}{\sqrt{P_{5}(s)}}, \theta_{2}=\frac{s d s}{\sqrt{P_{5}(s)}},
$$

and $P_{0}$ is a fixed point. Consequently, we have

Theorem 4.1. The system of differential equations (4.1) in the Clebsch's case can be integrated in terms of genus 2 hyperelliptic functions of time.

\subsection{Lyapunov-Steklov's case}

In Lyapunov-Steklov'case, we have

$$
H_{1}=H=\frac{1}{2} \sum_{k=1}^{3}\left(a_{k} p_{k}^{2}+b_{k} l_{k}^{2}\right)+\sum_{k=1}^{3} c_{k} p_{k} l_{k}
$$

with

$$
\begin{aligned}
& a_{1}=A^{2} b_{1}\left(b_{2}-b_{3}\right)^{2}+B, \quad a_{2}=A^{2} b_{2}\left(b_{3}-b_{1}\right)^{2}+B, \quad a_{3}=A^{2} b_{3}\left(b_{1}-b_{2}\right)^{2}+B, \\
& c_{1}=A b_{2} b_{3}+C, \quad c_{2}=A b_{1} b_{3}+C, \quad c_{3}=A b_{1} b_{2}+C,
\end{aligned}
$$

where $A, B, C$ are constants. A fourth first integral is given by

$$
H_{4}=\frac{1}{2} \sum_{k=1}^{3}\left(d_{k} p_{k}^{2}+l_{k}^{2}\right)-A \sum_{k=1}^{3} b_{k} p_{k} l_{k}
$$

where

$$
d_{1}=A^{2}\left(b_{2}-b_{3}\right)^{2}, \quad d_{2}=A^{2}\left(b_{3}-b_{1}\right)^{2}, \quad d_{3}=A^{2}\left(b_{1}-b_{2}\right)^{2} .
$$

A long and delicate calculation [20] shows that in this case too, the integration is done using hyperelliptic functions of genus two.

\section{The classification of algebraic integrable geodesic flow on $S O(4)$}

In several problems, , when studying the geodesic flow on $S O(4)$, it is more convenient to use the coordinates $u=\left(x_{1}, x_{2}, x_{3}\right)$ and $v=\left(x_{4}, x_{5}, x_{6}\right)$, they correspond to the decomposition $u \oplus v \in s o(4) \simeq \operatorname{so}(3) \oplus \operatorname{so}(3)$. In these coordinates, the geodesic flow on the group $S O(4)$ can be written as

$$
X_{H}: \dot{u}=u \wedge \frac{\partial H}{\partial u}, \dot{v}=v \wedge \frac{\partial H}{\partial v}
$$


for invariant metric defined by the quadratic form

$$
H=\frac{1}{24} \sum_{i=1}^{3}\left(3\left(3 c_{i}+d_{i}\right) x_{i}^{2}+\left(c_{i}+3 d_{i}\right) x_{i+3}^{2}+6\left(d_{i}-c_{i}\right) x_{i} x_{i+3}\right)
$$

with coefficients

$$
c_{i}=\frac{b_{i}}{a_{i}}, d_{i}=\frac{b_{j}-b_{k}}{a_{j}-a_{k}}, \sum_{i=1}^{3} a_{i}=0, \sum_{i=1}^{3} b_{i}=0,
$$

and $i j k$ permutations of 123 . This geodesic flow has three quadratic invariants, namely, the Casimir functions $\|u\|^{2}$ and $\|v\|^{2}$, and the metric above, and one quartic invariant. The invariants $\|u\|^{2}$ and $\|v\|^{2}$ define the 4-dimensional non degenerate symplectic leaves of Hamiltonian structure, which therefore are parameterized by the values of $\|u\|^{2}$ and $\|v\|^{2}$. More precisely, in the classification $[4,6]$ of algebraic integrable geodesic flow on $S O(4)$, three cases come up; two are linearly equivalent to cases of rigid body motion in a perfect fluid studied last century, respectively by Clebsch and Lyapunov-Steklov, and there is a third new case namely the Kostant-Kirillov Hamiltonian flow on the dual of $s o(4)$. The metric $H$ is obviously written in the quadratic form

$$
H=\frac{1}{2} \sum_{j=1}^{6} \lambda_{j} x_{j}^{2}+\sum_{j=1}^{3} \mu_{j} x_{j} x_{j+3}
$$

where $\lambda_{1}, \ldots, \lambda_{6}, \mu_{1}, \mu_{2}, \mu_{3} \in \mathbb{C}$ and $\lambda_{12} \lambda_{23} \lambda_{31} \lambda_{45} \lambda_{56} \lambda_{64} \mu_{1} \mu_{2} \mu_{3} \neq 0$ with $\lambda_{j k} \equiv \lambda_{j}-\lambda_{k}$. Explicitly, the equations above are written

$$
\begin{aligned}
\frac{d x_{1}}{d t} & =\lambda_{32} x_{2} x_{3}+\mu_{3} x_{2} x_{6}-\mu_{2} x_{3} x_{5}, \\
\frac{d x_{2}}{d t} & =\lambda_{13} x_{3} x_{1}+\mu_{1} x_{3} x_{4}-\mu_{3} x_{1} x_{5}, \\
\frac{d x_{3}}{d t} & =\lambda_{21} x_{1} x_{2}+\mu_{2} x_{1} x_{5}-\mu_{1} x_{2} x_{4}, \\
\frac{d x_{4}}{d t} & =\lambda_{65} x_{5} x_{6}+\mu_{3} x_{3} x_{5}-\mu_{2} x_{2} x_{6}, \\
\frac{d x_{5}}{d t} & =\lambda_{46} x_{6} x_{4}+\mu_{1} x_{1} x_{6}-\mu_{3} x_{3} x_{4}, \\
\frac{d x_{6}}{d t} & =\lambda_{54} x_{4} x_{5}+\mu_{2} x_{2} x_{4}-\mu_{1} x_{1} x_{5} .
\end{aligned}
$$

The equations have besides the energy $H_{1}=H$, two trivial constants of the motion

$$
H_{2}=x_{1}^{2}+x_{2}^{2}+x_{3}^{2}, H_{3}=x_{4}^{2}+x_{5}^{2}+x_{6}^{2} .
$$

Adler and van Moerbeke $[4,6]$ have shown that the geodesic flow on $S O(4)$ for the metric defined by the above quadratic form is algebraically completely integrable in the three cases described in the following subsections and these are the only ones that exist.

\subsection{Manakov geodesic flow on the group $S O(4)$ and Clebsch rigid body motion in a perfect fluid} The geodesic flow for this metric takes the following commutator form (Euler-Arnold equations) :

$$
\dot{X}=[X, \Lambda . X], \quad \cdot \equiv \frac{d}{d t}
$$

where

$$
X=\left(X_{i j}\right)_{1 \leq i, j \leq 4}=\sum_{i=1}^{6} x_{i} e_{i}=\left(\begin{array}{cccc}
0 & -x_{3} & x_{2} & -x_{4} \\
x_{3} & 0 & -x_{1} & -x_{5} \\
-x_{2} & x_{1} & 0 & -x_{6} \\
x_{4} & x_{5} & x_{6} & 0
\end{array}\right) \in \operatorname{so}(4) \text {, }
$$


and

$$
\Lambda . X=\left(\lambda_{i j} X_{i j}\right)_{1 \leq i, j \leq 4}=\sum_{i=1}^{6} \lambda_{i} x_{i} e_{i}=\left(\begin{array}{cccc}
0 & -\lambda_{3} x_{3} & \lambda_{2} x_{2} & -\lambda_{4} x_{4} \\
\lambda_{3} x_{3} & 0 & -\lambda_{1} x_{1} & -\lambda_{5} x_{5} \\
-\lambda_{2} x_{2} & \lambda_{1} x_{1} & 0 & -\lambda_{6} x_{6} \\
\lambda_{4} x_{4} & \lambda_{5} x_{5} & \lambda_{6} x_{6} & 0
\end{array}\right) \in \operatorname{so}(4)
$$

In view of the isomorphism between $\left(\mathbb{R}^{6}, \wedge\right)$ and $(s o(4),[]$,$) we write the system (5.2) as$

$$
\begin{aligned}
& \dot{x}_{1}=\left(\lambda_{3}-\lambda_{2}\right) x_{2} x_{3}+\left(\lambda_{6}-\lambda_{5}\right) x_{5} x_{6}, \\
& \dot{x}_{2}=\left(\lambda_{1}-\lambda_{3}\right) x_{1} x_{3}+\left(\lambda_{4}-\lambda_{4}\right) x_{4} x_{6}, \\
& \dot{x}_{3}=\left(\lambda_{2}-\lambda_{1}\right) x_{1} x_{2}+\left(\lambda_{5}-\lambda_{4}\right) x_{4} x_{5}, \\
& \dot{x}_{4}=\left(\lambda_{3}-\lambda_{5}\right) x_{3} x_{5}+\left(\lambda_{6}-\lambda_{2}\right) x_{2} x_{6}, \\
& \dot{x}_{5}=\left(\lambda_{4}-\lambda_{3}\right) x_{3} x_{4}+\left(\lambda_{1}-\lambda_{6}\right) x_{1} x_{6}, \\
& \dot{x}_{6}=\left(\lambda_{2}-\lambda_{4}\right) x_{2} x_{4}+\left(\lambda_{5}-\lambda_{1}\right) x_{1} x_{5} .
\end{aligned}
$$

The quadratic form $H$ is diagonal with regard to the customary so(4) coordinates (Manakov metric [29]), i.e.,

$$
\left.2 H=\sum_{\substack{j, k=1 \\ j<k}}^{4} \Lambda_{j k} X_{j k}^{2}, \quad\left(X_{j k}\right)_{1 \leq j, k \leq 4}\right) \in \operatorname{so}(4),
$$

with

$$
\Lambda_{j k}=\frac{\beta_{j}-\beta_{k}}{\alpha_{j}-\alpha_{k}}, \quad\left(\alpha_{j}, \beta_{j} \in \mathbb{C}, 1 \leq j \leq 4\right),
$$

all $\Lambda_{j k}$ distinct. The extra invariant $H_{4}$ is quadratic and we'll see how this was done independently by Haine [13] and Mumford (appendix in[2]) that the flow evolves on Abelian surfaces $\mathbb{C}^{2} /$ lattice $\subseteq \mathbb{P}^{7}(\mathbb{C})$, having period matrix

$$
\left(\begin{array}{llll}
2 & 0 & a & c \\
0 & 4 & c & b
\end{array}\right), \quad \operatorname{Im}\left(\begin{array}{ll}
a & c \\
c & b
\end{array}\right)>0, \quad(a, b, c \in \mathbb{C}),
$$

and also the linearization takes place on a Prym variety (For the definition and properties of Prym varieties, see for example $[13,25])$. The periods of this Prym variety provide the exact periods of the motion in terms of Abelian integrals. The problem of the solid body in a fluid in the case of Clebsch is a particular case of this metric (see subsection 4.1).

Let's see in more detail the linearization of this problem $[13,6,22]$. Let $x \in \mathbb{C}^{6}, t \in \mathbb{C}$ and $Z \subset \mathbb{C}^{6}$ a non-empty Zariski open set. The momentum map

$$
g:\left(H_{1}, \ldots, H_{4}\right): \mathbb{C}^{6} \longrightarrow \mathbb{C}^{4},
$$

is submersive on $Z$, i.e., $d H_{1}(x), \ldots, d H_{4}(x)$ are linearly independent on $Z$. Let

$$
I=g\left(\mathbb{C}^{6} \backslash Z\right)=\left\{c=\left(c_{i}\right) \in \mathbb{C}^{4}: \exists x \in g^{-1}(c) \text { with } d H_{1}(x) \wedge \ldots \wedge d H_{4}(x)=0\right\},
$$

be the set of critical values of $g$ and $\bar{I}$ the Zariski closure of $I$ in $\mathbb{C}^{4}$. The non-empty Zariski open set $Z$ can be chosen as the set

$$
Z=\left\{x \in \mathbb{C}^{6}: g(x) \in \mathbb{C}^{4} \backslash \bar{I}\right\} .
$$

The invariant variety defined by

$$
M_{c}=g^{-1}(c)=\bigcap_{i=1}^{4}\left\{x \in \mathbb{C}^{6}: H_{i}(x)=c_{i}\right\},
$$

is the fibre of a morphism from $\mathbb{C}^{6}$ to $\mathbb{C}^{4}$, thus $M_{c}$ is a smooth affine surface for generic $c=\left(c_{1}, \ldots, c_{4}\right) \in \mathbb{C}^{4}$ and the main problem will be to complete $M_{c}$ into an Abelian surface. Now, how does one find the compactification of $M_{c}$ into an Abelian surface? This compactification is not trivial and the simplest one obtained as a closure :

$$
\overline{M_{c}}=\bigcap_{i=1}^{4}\left\{H_{i}(x)=c_{i} x_{0}^{2}\right\} \subset \mathbb{P}^{6}(\mathbb{C}),
$$


i.e.,

$$
\begin{aligned}
x_{1} x_{4}+x_{2} x_{5}+x_{3} x_{6} & =c_{1} x_{0}^{2}, \\
x_{1}^{2}+x_{2}^{2}+\cdots+x_{6}^{2} & =c_{2} x_{0}^{2}, \\
\lambda_{1} x_{1}^{2}+\lambda_{2} x_{2}^{2}+\cdots+\lambda_{6} x_{6}^{2} & =c_{3} x_{0}^{2}, \\
\mu_{1} x_{1}^{2}+\mu_{2} x_{2}^{2}+\cdots+\mu_{6} x_{6}^{2} & =c_{4} x_{0}^{2},
\end{aligned}
$$

where $\left[x_{0}: x_{1}: \ldots: x_{6}\right]$ are homogeneous coordinates on $\mathbb{P}^{6}(\mathbb{C})$, does not lead to this result (in the following we will not distinguish between $x_{1}$ as a homogeneous coordinates $\left[x_{0}: x_{1}\right]$ and as an affine coordinate $\left.x_{1} / x_{0}\right)$. Indeed, an Abelian surface is not simply-connected and therefore cannot be projective complete intersection. In other words, if $M_{c}$ is to be the affine part of an Abelian surface, $M_{c}$ must have a singularity somewhere along the locus at infinity $C=\overline{M_{c}} \cap\left\{x_{0}=0\right\}$. A direct calculation shows that $C$ is an ordinary double curve of $M_{c}$ except at 16 ordinary pinch points of $\overline{M_{c}}$; the variety $\overline{M_{c}}$ has a local analytic equation $x^{2}=y z^{2}$. The reduced curve $C_{r}$ is a smooth elliptic curve. Now, it's only after blowing up $\overline{M_{c}}$ along the curve $C_{r}$ that one gets the desired Abelian surface.

Theorem 5.1. a) The divisor of poles of the functions $x_{1}, x_{2}, \ldots, x_{6}$ is a Riemann surface $\mathscr{D}$ of genus 9 . For generic constants, the surface $M_{c}$ is the affine part of an Abelian surface $\widetilde{M}_{c}$ obtained by gluing to $M_{c}$ the divisor $\mathscr{D}$.

b) The flow (5.3) evolves on an Abelian surface $\widetilde{M}_{c} \cong \mathbb{C}^{2} /$ lattice of polarization

$$
\left(\begin{array}{llll}
2 & 0 & a & c \\
0 & 4 & c & b
\end{array}\right), \operatorname{Im}\left(\begin{array}{ll}
a & c \\
c & b
\end{array}\right)>0
$$

c) The Abelian surface $\widetilde{M}_{c}$ which completes the affine surface $M_{c}$ is the Prym variety Prym ${ }_{\alpha}(\Gamma)$ of the genus 3 Riemann surface $\Gamma$ :

$$
\Gamma:\left\{\begin{array}{l}
w^{2}=-c_{1}\left(x_{5}^{0} x_{6}^{0}\right)^{2}-c_{2}\left(x_{6}^{0}\right)^{2} z-c_{3}\left(x_{5}^{0}\right)^{2} z+c_{4} y \\
y^{2}=z\left(\alpha^{2} z-1\right)\left(\beta^{2} z+1\right)
\end{array}\right.
$$

for the involution

$$
\sigma: \Gamma \longrightarrow \Gamma,(w, y, z) \longmapsto(-w, y, z),
$$

interchanging the two sheets of the double covering

$$
\Gamma \longmapsto \Gamma_{0}, \quad(w, y, z) \longmapsto(y, z),
$$

where $\Gamma_{0}$ is the elliptic curve defined by

$$
\Gamma_{0}: y^{2}=z\left(\alpha^{2} z-1\right)\left(\beta^{2} z+1\right) \text {. }
$$

Proof. a) Consider points at infinity which are limit points of trajectories of the flow. There is a Laurent decomposition of such asymptotic solutions,

$$
X(t)=t^{-1}\left(X^{(0)}+X^{(1)} t+X^{(2)} t^{2}+\cdots\right)
$$

which depend on $\operatorname{dim}$ (phase space) $-1=5$ free parameters. Putting (5.4) into (5.2), solving inductively for the $X^{(k)}$, one finds at the $0^{\text {th }}$ step a non-linear equation,

$$
X^{(0)}+\left[X^{(0)}, \Lambda . X^{(0)}\right]=0,
$$

and at the $k^{\text {th }}$ step, a linear system of equations

$$
(L-k I)\left(X^{(k)}\right)=\left\{\begin{array}{rr}
0 & \text { for } k=1 \\
\text { quadratic polynomial in } X^{(1)}, \ldots, X^{(k-1)} & \text { for } k \geq 2
\end{array}\right.
$$

where $L$ denotes the linear map

$$
L(Y)=\left[Y, \Lambda . X^{(0)}\right]+\left[X^{(0)}, \Lambda . Y\right]+Y=\text { Jacobian map of (5.5). }
$$


One parameter appear at the $0^{\text {th }}$ step, i.e., in the resolution of (5.5) and the 4 remaining ones at the $k^{\text {th }}$ step, $k=1, \ldots, 4$. Taking into account only solutions trajectories lying on the surface $M_{c}$, we obtain one-parameter families which are parameterized by a Riemann surface. To be precise we search for the set $\mathscr{D}$ of Laurent solutions (5.4) restricted to the affine invariant surface $M_{c}$, i.e.,

$$
\begin{aligned}
\mathscr{D}= & \text { closure of the continuous components of } \\
& \left\{\text { Laurent solutions } X(t) \text { such that } H_{i}(X(t))=c_{i}, 1 \leq i \leq 4\right\}, \\
= & \bigcap_{i=1}^{4}\left\{t^{0}-\text { coefficient of } H_{i}(X(t))=c_{i}\right\}, \\
= & \text { a Riemann surface (algebraic curve) whose affine equation is } \\
& \left\{\begin{array}{c}
w^{2}+c_{1}\left(x_{5}^{(0)} x_{6}^{(0)}\right)^{2}+c_{2}\left(x_{4}^{(0)} x_{6}^{(0)}\right)^{2}+c_{3}\left(x_{4}^{(0)} x_{5}^{(0)}\right)^{2}+c_{4} x_{4}^{(0)} x_{5}^{(0)} x_{6}^{(0)}, \\
\equiv w^{2}+F\left(x_{4}^{(0)}, x_{5}^{(0)}, x_{6}^{(0)}\right)=0
\end{array}\right.
\end{aligned}
$$

where $w$ is an arbitrary parameter and where $x_{4}^{(0)}, x_{5}^{(0)}, x_{6}^{(0)}$ parameterizes the elliptic curve

$$
\mathscr{E}:\left\{\begin{aligned}
\left(x_{4}^{(0)}\right)^{2}+\left(x_{5}^{(0)}\right)^{2}+\left(x_{6}^{(0)}\right)^{2}=0 \\
\left(\beta x_{5}^{(0)}+\alpha x_{6}^{(0)}\right)\left(\beta x_{5}^{(0)}-\alpha x_{6}^{(0)}\right)=1
\end{aligned}\right.
$$

with $(\alpha, \beta)$ such that : $\alpha^{2}+\beta^{2}+1=0$. The Riemann surface $\mathscr{D}$ is a two-sheeted ramified covering of the elliptic curve $\mathscr{E}$ and it easy to check that the elliptic curve $\mathscr{E}$ is exactly the reduced curve $C_{r}$. The branch points are defined by the 16 zeroes of $F\left(x_{4}^{(0)}, x_{5}^{(0)}, x_{6}^{(0)}\right)$ on $\mathscr{E}$. The Riemann surface $\mathscr{D}$ is unramified at infinity and by Riemann-Hurwitz's formula,

$$
2 g(\mathscr{D})-2=N(2 g(\mathscr{E})-2)+R,
$$

where $N$ is the number of sheets and $R$ the ramification index, the genus $g(\mathscr{D})$ of $\mathscr{D}$ is 9 . To show that $M_{c}$ is the affine part of an Abelian surface $\widetilde{M}_{c}$ with $\widetilde{M}_{c} \backslash M_{c}=\mathscr{D}$, we can use the method of Laurent's developments (see Haine [13]) or theorem 2.3. Here, by following Mumford (see appendix to [2]), we give an abstract algebro-geometrical proof that the four quadrics in this problem intersect in the affine part of an Abelian surface using Enriques classification of algebraic surfaces. For this, we will compute the invariants of $\widetilde{M}_{c}$ and use Enriques classification of algebraic surfaces (see [12], p.590). We denote as usual by $K_{\widetilde{M}_{c}}$ the canonical bundle, $\chi\left(\mathscr{O}_{\widetilde{M}_{c}}\right)$ the Euler characteristic and $q\left(\widetilde{M}_{c}\right)$ the irregularity of $\widetilde{M_{c}}$. Now if

$$
\phi: \widetilde{M_{c}} \longrightarrow \overline{M_{c}} \subset \mathbb{P}^{6}(\mathbb{C})
$$

is the normalization of $\overline{M_{c}}$, then the pullback map on sections

$$
\phi^{*}: H^{0}\left(\overline{M_{c}}, \mathscr{O}_{\overline{M_{c}}}\right) \longrightarrow H^{0}\left(\widetilde{M}_{c}, \mathscr{O}_{\widetilde{M}_{c}}\right),
$$

is an isomorphism and

$$
K_{\widetilde{M_{c}}}=\widetilde{K_{\overline{M_{c}}}}-\mathscr{D}, \quad \widetilde{K_{\overline{M_{c}}}}=\phi^{*}\left(K_{\overline{M_{c}}}\right)
$$

and so for $H$ a hyperplane in $\mathbb{P}^{6}(\mathbb{C})$,

$$
K_{\widetilde{M}_{c}}=\phi^{*}\left(\overline{M_{c}} \cdot K_{\mathbb{P} 6(\mathbb{C})}+\left(\sum_{i=1}^{4} \operatorname{deg} H_{i}\right) \cdot H\right)-\mathscr{D}=0 .
$$

Also

$$
\chi\left(\mathscr{O}_{\widetilde{M_{c}}}\right)=\chi\left(\phi_{*} \mathscr{O}_{\widetilde{M}_{c}} / \mathscr{O}_{\overline{M_{c}}}\right)+\chi\left(\mathscr{O}_{\overline{M_{c}}}\right)=\chi\left(\phi_{*} \mathscr{O}_{\mathscr{D}} / \mathscr{O}_{\mathscr{E}}\right)+\chi\left(\mathscr{O}_{\overline{\mathscr{A}}}\right) .
$$

Recall that the Riemann surface $\mathscr{D}$ (5.7) of genus 9, is a double cover ramified over 16 points of the elliptic curve $\mathscr{E}(5.8)$. We shall use the Koszul complex to compute $\chi\left(\mathscr{O}_{\overline{M_{c}}}\right)$. In the local ring at each point of $\mathbb{P}^{6}(\mathbb{C})$ the localizations of the 4 homogeneous polynomials $H_{i}$ give a regular sequence, and the Koszul complex gives a canonical resolution

$$
0 \rightarrow \mathscr{O}_{\mathbb{P}^{6}(\mathbb{C})}(-8) \rightarrow \mathscr{O}_{\mathbb{P}^{6}(\mathbb{C})}(-6)^{4} \rightarrow \mathscr{O}_{\mathbb{P}^{6}(\mathbb{C})}(-4)^{6} \rightarrow \mathscr{O}_{\mathbb{P}^{6}(\mathbb{C})}(-2)^{4} \rightarrow \mathscr{O}_{\mathbb{P}^{6}(\mathbb{C})} \rightarrow \mathscr{O}_{\bar{M}_{c}} \rightarrow 0
$$


Thus $\chi\left(\mathscr{O}_{\overline{M_{c}}}\right)=8$, hence $\chi\left(\mathscr{O}_{\widetilde{M}_{c}}\right)=0$ and $q\left(\widetilde{M}_{c}\right)=2$. By Enriques-Kodaira's classification theorem, it follows that $\widetilde{M}_{c}$ is an Abelian surface.

b) Let

$$
L \equiv\left\{f: f \text { meromorphic on } \widetilde{M}_{c},(f)+\mathscr{D} \geq 0\right\}
$$

be the set of meromorphic functions on $\widetilde{M}_{c}$ with at worst a simple pole along $\mathscr{D}$ and let

$$
\chi(\mathscr{D})=\operatorname{dim} H^{0}\left(\widetilde{M}_{c}, \mathscr{O}(\mathscr{D})\right)-\operatorname{dim} H^{1}\left(\widetilde{M}_{c}, \mathscr{O}(\mathscr{D})\right),
$$

be the Euler characteristic of $\mathscr{D}$. The adjunction formula and the Riemann-Roch theorem for divisors on Abelian surfaces imply that

$$
g(\mathscr{D})=\frac{K_{\widetilde{M}_{c}} \cdot \mathscr{D}+\mathscr{D} \cdot \mathscr{D}}{2}+1
$$

and

$$
\chi(\mathscr{D})=p_{a}\left(\widetilde{M}_{c}\right)+1+\frac{1}{2}\left(\mathscr{D} \cdot\left(\mathscr{D}-K_{\widetilde{M}_{c}}\right)\right),
$$

where $g(\mathscr{D})$ is the geometric genus of $\mathscr{D}$ and $p_{a}\left(\widetilde{M}_{c}\right)$ is the arithmetic genus of $\widetilde{M}_{c}$. Since $\widetilde{M}_{c}$ is an Abelian surface

$$
\begin{aligned}
\left(K_{\widetilde{M}_{c}}=0, p_{a}\left(\widetilde{M}_{c}\right)=-1\right), \\
\quad g(\mathscr{D})-1=\frac{\mathscr{D} \cdot \mathscr{D}}{2}=\chi(\mathscr{D}) .
\end{aligned}
$$

Using Kodaira-Serre duality [12, 27], Kodaira-Nakano vanishing theorem [12, 27] and a theorem on theta-functions [12, 27], it easy to see that

$$
g(\mathscr{D})-1=\operatorname{dim} L(\mathscr{D})\left(\equiv h^{0}(L)\right)=\delta_{1} \delta_{2},
$$

where $\delta_{1}, \delta_{2} \in \mathbb{N}$, are the elementary divisors of the polarization $c_{1}(L)$ of $\widetilde{M}_{c}$. Note that the natural reflection about the origin of $\mathbb{C}^{2}$, is given by

$$
\sigma \equiv-i d:\left(x_{0}, x_{1}, \ldots, x_{6}\right) \longmapsto\left(-x_{0}, x_{1}, \ldots, x_{6}\right),
$$

and has 16 fixed points on $\widetilde{M}_{c}$, given by the 16 branch points on $\mathscr{D}$ covering the 16 roots of the polynomial $F\left(x_{4}^{0}, x_{5}^{0}, x_{6}^{0}\right)(5.7)$. Since $L$ is symmetric $\left(\sigma^{*} L \simeq L\right), \sigma$ can be lifted to $L$ as an involution $\widetilde{\sigma}$ in two ways differing in sign and for each section (theta-function) $s \in H^{0}(L)$, we therefore have $\widetilde{\sigma} s= \pm s$. Recall that a section $s \in H^{0}(L)$ is called even (resp. odd) if $\widetilde{\sigma} s=+s$ (resp. $\widetilde{\sigma} s=-s$ ). Under $\widetilde{\sigma}$ the vector space $H^{0}(L)$ splits into an even and odd subspace $H^{0}(L)=H^{0}(L)^{\text {even }} \oplus H^{0}(L)^{\text {odd }}$ with $H^{0}(L)^{\text {even }}$ containing all the even sections and $H^{0}(L)^{\text {odd }}$ all odd ones. Using the inverse formula [12, 27], we see after a small computation that

$$
\begin{aligned}
\operatorname{dim} H^{0}(\mathscr{L})^{\text {even }} & =\frac{\delta_{1} \delta_{2}}{2}+2^{-1+\sharp \text { even } \delta_{k}}, k=1,2 \\
\operatorname{dim} H^{0}(\mathscr{L})^{\text {odd }} & =\frac{\delta_{1} \delta_{2}}{2}-2^{-1+\sharp \text { even } \delta_{k}}, k=1,2
\end{aligned}
$$

Notice that $c_{1}(L)=\phi^{*}(H)$ and $\left(c_{1}(L)^{2}\right)=16$ (since the degree of $\overline{M_{c}}$ is 16). By the classification theory of ample line bundles on Abelian varieties, $\widetilde{M}_{c} \simeq \mathbb{C}^{2} / L_{\Omega}$ with period lattice given by the columns of the matrix

$$
\Omega=\left(\begin{array}{cccc}
\delta_{1} & 0 & a & c \\
0 & \delta_{2} & c & b
\end{array}\right), \operatorname{Im}\left(\begin{array}{cc}
a & c \\
c & b
\end{array}\right)>0
$$

according to (5.9), with

$$
\delta_{1} \delta_{2}=h^{0}(L)=g(\mathscr{D})-1=8, \quad \delta_{1} \mid \delta_{2}, \quad \delta_{i} \in \mathbb{N}^{*} .
$$


Hence we have two possibilities : ( $i) \delta_{1}=1, \delta_{2}=8$ and (ii) $\delta_{1}=2, \delta_{2}=4$. From formula (5.10), the corresponding line bundle $L$ has in case $(i), 5$ even sections, 3 odd ones and in case $(i i), 6$ even sections, 2 odd ones. Now $x_{1}, \ldots, x_{6}$ are 6 even sections, showing that case (ii) is the only alternative and the period matrix has the form

$$
\left(\begin{array}{llll}
2 & 0 & a & c \\
0 & 4 & c & b
\end{array}\right), \operatorname{Im}\left(\begin{array}{ll}
a & c \\
c & b
\end{array}\right)>0
$$

c) After substitution $z \equiv\left(x_{4}^{0}\right)^{2}$, the Riemann surface $\mathscr{D}$ can also be seen as a four-sheeted unramified covering of another Riemann surface $\Gamma$, determined by the equation

$$
\Gamma: G(w, z) \equiv\left(w^{2}+c_{1}\left(x_{5}^{0} x_{6}^{0}\right)^{2}+c_{2}\left(x_{6}^{0}\right)^{2} z+c_{3}\left(x_{5}^{0}\right)^{2} z\right)^{2}-c_{4}^{2}\left(x_{5}^{0} x_{6}^{0}\right)^{2} z=0 .
$$

It is straightforward to verify that the equations (5.8) are equivalent to $\left(x_{5}^{0}\right)^{2}=\beta^{2} z+1$ and $\left(x_{6}^{0}\right)^{2}=\alpha^{2} z-1$. To compute the genus of $\Gamma$, we observe that the Riemann surface $\Gamma$ is invariant under an involution

$$
\sigma: \Gamma \longrightarrow \Gamma, \quad(w, z) \longmapsto(-w, z) .
$$

Consider a map

$$
\rho: \Gamma \longrightarrow \Gamma_{0} \equiv \Gamma / \sigma, \quad(w, y, z) \longmapsto(y, z),
$$

of the Riemann surface $\Gamma$ onto an elliptic curve $\Gamma_{0} \equiv \Gamma / \sigma$, that is given by the equation

$$
\Gamma_{0}: y^{2}=z\left(\alpha^{2} z-1\right)\left(\beta^{2} z+1\right) .
$$

The genus of the Riemann surface

$$
\Gamma:\left\{\begin{array}{l}
w^{2}=-c_{1}\left(x_{5}^{0} x_{6}^{0}\right)^{2}-c_{2}\left(x_{6}^{0}\right)^{2} z-c_{3}\left(x_{5}^{0}\right)^{2} z+c_{4} y \\
y^{2}=z\left(\alpha^{2} z-1\right)\left(\beta^{2} z+1\right)
\end{array}\right.
$$

is calculated by means of the map $\rho$. The latter is two-sheeted ramified covering of the elliptic curve $\Gamma_{0}$ with 4 branch points. Using the Riemann-Hurwitz formula, we obtain $g(\Gamma)=3$.

I now will proceed to show that the Abelian surface $\widetilde{M}_{c}$ can be identified as Prym variety $\operatorname{Prym}_{\sigma}(\Gamma)$. Let $\left(a_{1}, a_{2}, a_{3}, b_{1}, b_{2}, b_{3}\right)$ be a basis of cycles in the Riemann surface $\Gamma$ with the intersection indices $a_{i} o a_{j}=b_{i} o b_{j}=0, a_{i} o b_{j}=\delta_{i j}$, such that

$$
\sigma\left(a_{1}\right)=a_{3}, \quad \sigma\left(b_{1}\right)=b_{3}, \quad \sigma\left(a_{2}\right)=-a_{2}, \quad \sigma\left(b_{2}\right)=-b_{2}
$$

for the involution $\sigma$ (5.11). By the Poincaré residue formula, the 3 holomorphic 1 -forms $\omega_{0}, \omega_{1}, \omega_{2}$ in $\Gamma$ are the differentials

$$
\left.P(w, z) \frac{d z}{(\partial G / \partial w)(w, z)}\right|_{G(w, z)=0}=P(w, z) \frac{d z}{4 w y},
$$

for $P$ a polynomial of degree $\leq \operatorname{deg} G-3=1$. Therefore

$$
\omega_{0}=\frac{d z}{y}, \omega_{1}=\frac{z d z}{w y}, \omega_{2}=\frac{d z}{w y},
$$

form a basis of holomorphic differentials on $\Gamma$ and obviously

$$
\sigma^{*}\left(\omega_{0}\right)=\omega_{0}, \sigma^{*}\left(\omega_{k}\right)=-\omega_{k}, \quad(k=1,2),
$$

for the involution $\sigma$ (5.11). It is well known that the period matrix $\Omega$ of $\operatorname{Prym}_{\sigma}(\Gamma)$ can be written as follows

$$
\Omega=\left(\begin{array}{cccc}
2 \int_{a_{1}} \omega_{1} & \int_{a_{2}} \omega_{1} & 2 \int_{b_{1}} \omega_{1} & \int_{b_{2}} \omega_{1} \\
2 \int_{a_{1}} \omega_{2} & \int_{a_{2}} \omega_{2} & 2 \int_{b_{1}} \omega_{2} & \int_{b_{2}} \omega_{2}
\end{array}\right),
$$

Let $\left(d t_{1}, d t_{2}\right)$ be a basis of holomorphic 1-forms on $\widetilde{M}_{c}$ such that $\left.d t_{j}\right|_{\mathscr{D}}=\omega_{j},(j=1,2)$,

$$
L_{\Omega^{\prime}}=\left\{\sum_{k=1}^{2} m_{k} \int_{a_{k}^{\prime}}\left(\begin{array}{l}
d t_{1} \\
d t_{2}
\end{array}\right)+n_{k} \int_{b_{k}^{\prime}}\left(\begin{array}{l}
d t_{1} \\
d t_{2}
\end{array}\right): m_{k}, n_{k} \in \mathbb{Z}\right\},
$$


the lattice associated to the period matrix

$$
\Omega^{\prime}=\left(\begin{array}{cccc}
\int_{a_{1}^{\prime}} d t_{1} & \int_{a_{2}^{\prime}} d t_{1} & \int_{b_{1}^{\prime}} d t_{1} & \int_{b_{2}^{\prime}} d t_{1} \\
\int_{a_{1}^{\prime}} d t_{2} & \int_{a_{2}^{\prime}} d t_{2} & \int_{b_{1}^{\prime}} d t_{2} & \int_{b_{2}^{\prime}} d t_{2}
\end{array}\right),
$$

where $\left(a_{1}^{\prime}, a_{2}^{\prime}, b_{1}^{\prime}, b_{2}^{\prime}\right)$ is a basis of $H_{1}\left(\widetilde{M_{c}}, \mathbb{Z}\right)$ and let

$$
\widetilde{M}_{c} \longrightarrow \mathbb{C}^{2} / L_{\Omega^{\prime}}: p \longmapsto \int_{p_{0}}^{p}\left(\begin{array}{l}
d t_{1} \\
d t_{2}
\end{array}\right)
$$

be the uniformizing map. By the Lefschetz theorem on hyperplane section [12, 27], the map $H_{1}(\mathscr{D}, \mathbb{Z}) \longrightarrow H_{1}\left(\widetilde{M_{c}}, \mathbb{Z}\right)$ induced by the inclusion $\mathscr{D} \hookrightarrow \widetilde{M}_{c}$ is surjective and consequently we can find 4 cycles $a_{1}^{\prime}, a_{2}^{\prime}, b_{1}^{\prime}, b_{2}^{\prime}$ on the Riemann surface $\mathscr{D}$ such that

$$
\Omega^{\prime}=\left(\begin{array}{cccc}
\int_{a_{1}^{\prime}} \omega_{1} & \int_{a_{2}^{\prime}} \omega_{1} & \int_{b_{3}^{\prime}} \omega_{1} & \int_{b_{4}^{\prime}} \omega_{1} \\
\int_{a_{1}^{\prime}} \omega_{2} & \int_{a_{2}^{\prime}} \omega_{2} & \int_{b_{3}^{\prime}} \omega_{2} & \int_{b_{4}^{\prime}} \omega_{2}
\end{array}\right),
$$

and

$$
L_{\Omega^{\prime}}=\left\{\sum_{k=1}^{2} m_{k} \int_{a_{k}^{\prime}}\left(\begin{array}{l}
\omega_{1} \\
\omega_{2}
\end{array}\right)+n_{k} \int_{b_{k}^{\prime}}\left(\begin{array}{l}
\omega_{1} \\
\omega_{2}
\end{array}\right): m_{k}, n_{k} \in \mathbb{Z}\right\} .
$$

Recalling that $F\left(x_{4}^{0}, x_{5}^{0}, x_{6}^{0}\right)(5.7)$ has 4 zeroes on $\Gamma_{0}$ (5.12) and 16 zeroes on $\mathscr{E}$ (5.8), it follows that the 4 cycles $a_{1}^{\prime}, a_{2}^{\prime}, b_{1}^{\prime}, b_{2}^{\prime}$ on $\mathscr{D}$ which we look for are $2 a_{1}, a_{2}, 2 b_{1}, b_{2}$ and they form a basis of $H_{1}\left(\widetilde{M_{c}}, \mathbb{Z}\right)$ such that

$$
\Omega^{\prime}=\left(\begin{array}{cccc}
2 \int_{a_{1}} \omega_{1} & \int_{a_{2}} \omega_{1} & 2 \int_{b_{1}} \omega_{1} & \int_{b_{2}} \omega_{1} \\
2 \int_{a_{1}} \omega_{2} & \int_{a_{2}} \omega_{2} & 2 \int_{b_{1}} \omega_{2} & \int_{b_{2}} \omega_{2}
\end{array}\right)=\Omega
$$

is a Riemann matrix. Thus, $\widetilde{M}_{c}$ and $\operatorname{Prym}_{\sigma}(\Gamma)$ are two Abelian varieties analytically isomorphic to the same complex torus $\mathbb{C}^{2} / L_{\Omega}$. By Chow's theorem $[12,27], \widetilde{M}_{c}$ and $\operatorname{Prym}_{\sigma}(\Gamma)$ are then algebraically isomorphic. This completes the proof of the theorem.

Remark 5.2. It is well known that this problem has been solved via the Lax spectral curve technique by Adler and van Moerbeke [1]. Strange as it may seem, the use of the spectral curve method may not give the tori correctly, but perhaps with period doubling, in contrast with the statement that the correct tori would be obtained by the Kowalewski-Painleve analysis. This indicated a need for caution in interpretation of the result for tori calculated from the Lax spectral curve technique. A striking example of this phenomenon appears in the problem studied in this subsection. We know from [1, 22], that the linearization of the Euler-Arnold equations obtained using the isospectral deformation method (Lax technique) takes place on the Prym variety $\operatorname{Prym}_{\sigma}(\mathscr{C})$ of a genus 3 Riemann surface $\mathscr{C}$; the latter is a double ramified cover of an elliptic curve $\mathscr{C}_{0}$. Also, we have just seen from the asymptotic analysis of the Euler-Arnold equations, the affine variety $M_{c}$ completes into an Abelian surface $\widetilde{M}_{c}$ upon adding a Riemann surface $\mathscr{D}$ of genus 9 , which is a 4-fold unramified cover of another Riemann surface $\Gamma$ of genus 3; the latter is a double ramified cover of an elliptic curve $\Gamma_{0}$. The Abelian surface $\widetilde{M}_{c}$ can also be identified as the Prym variety $\operatorname{Prym}_{\sigma}(\Gamma)$ and the problem linearizes on $\operatorname{Prym}_{\sigma}(\Gamma)$. From the fundamental exponential sequence

$$
0 \rightarrow \mathbb{Z} \rightarrow \mathscr{O}_{\widetilde{M}_{c}} \stackrel{\exp }{\rightarrow} \mathscr{O}_{\widetilde{M}_{c}}^{*} \rightarrow 0,
$$

we get the map

$$
\cdots \rightarrow H^{1}\left(\widetilde{M}_{c}, \mathscr{O}_{\widetilde{M}_{c}}^{*}\right) \rightarrow H^{2}\left(\widetilde{M}_{c}, \mathbb{Z}\right) \rightarrow \cdots
$$

i.e., the first Chern class of a line bundle on $\widetilde{M}_{c}$. Recall that any line bundle with Chern class zero can be realized by constant multipliers. Therefore the group Pic ${ }^{o}\left(\widetilde{M}_{c}\right)$ of holomorphic line bundles on $\widetilde{M}_{c}$ with Chern class zero is given by

$$
\operatorname{Pic}^{o}\left(\widetilde{M}_{c}\right)=H^{1}\left(\widetilde{M}_{c}, \mathscr{O}_{\widetilde{M}_{c}}\right) / H^{1}\left(\widetilde{M}_{c}, \mathbb{Z}\right),
$$

and is naturally isomorphic to the dual Abelian surface $\widetilde{M}_{c}^{\vee}$ of $\widetilde{M}_{c}(\vee$ means the dual Abelian surface). The relationship between $\widetilde{M}_{c}$ and $\widetilde{M}_{c}^{\vee}$ is symmetric like the relationship between two vectors spaces set up a bilinear pairing. It is interesting 
to observe that the Abelian surfaces $\widetilde{M_{c}}=\operatorname{Prym}_{\sigma}(\Gamma)$ obtained from the asymptotic analysis of the differential equations and $\operatorname{Prym}_{\sigma}(\mathscr{C})$ obtained from the orbits in the Kac-Moody Lie algebra are not identical but only isogenous, i.e., one can be obtained from the other by doubling some periods and leaving other unchanged. The precise relation between these two Abelian surfaces is $\widetilde{M}_{c}=\left(\operatorname{Prym}_{\sigma}(\mathscr{C})\right)^{\vee}$, i.e., they are dual of each other. The functions $x_{1}, \ldots, x_{6}$ are themselves meromorphic on $\widetilde{M}_{c}$, while only their squares are on $\operatorname{Prym}_{\sigma}(\mathscr{C})$. The relationship between the Riemann surfaces $\Gamma$ and $\mathscr{C}$ is quite intricate. As usual we let $\Theta$ the theta divisor on $\operatorname{Jac}(\Gamma)$, we have

$$
\operatorname{Prym}_{\sigma}(\mathscr{C}) \backslash \Pi=\Theta \cap \operatorname{Prym}_{\sigma}(\mathscr{C})=\Gamma,
$$

with $\Pi$ a Zariski open set of $\operatorname{Prym}_{\sigma}(\mathscr{C})$. Also

$$
\Theta \cap \widetilde{M_{c}}=\mathscr{C}
$$

where $\Theta$ is a translate of the theta divisor of Jac $(\mathscr{C})$ invariant under the involution $\sigma$. Moser [31] was aware of a similar situation in the context of the Jacobi's geodesic flow problem on ellipsoids.

\subsection{Manakov geodesic flow on the group $\mathrm{SO}(4)$ and Lyapunov-Steklov rigid body motion in a perfect fluid} The quadratic form $H$ satisfies the conditions

$$
\left(\mu_{1}^{2}, \mu_{2}^{2}, \mu_{3}^{2}\right)=\frac{\lambda_{12} \lambda_{23} \lambda_{31} \lambda_{45} \lambda_{56} \lambda_{64}}{\left(\lambda_{46} \lambda_{32}-\lambda_{65} \lambda_{13}\right)^{2}}\left(\frac{\left(\lambda_{23}-\lambda_{56}\right)^{2}}{\lambda_{23} \lambda_{56}}, \frac{\left(\lambda_{31}-\lambda_{64}\right)^{2}}{\lambda_{31} \lambda_{64}}, \frac{\left(\lambda_{12}-\lambda_{45}\right)^{2}}{\lambda_{12} \lambda_{45}}\right),
$$

with the product $\mu_{1} \mu_{2} \mu_{3}$ being rational in $\lambda_{1}, \ldots, \lambda_{6}$ and with the following sign specification

$$
\mu_{1} \mu_{2} \mu_{3}=\frac{\lambda_{12} \lambda_{23} \lambda_{31} \lambda_{45} \lambda_{56} \lambda_{64}}{\left(\lambda_{46} \lambda_{32}-\lambda_{65} \lambda_{13}\right)^{3}}\left(\lambda_{12}-\lambda_{45}\right)\left(\lambda_{23}-\lambda_{56}\right)\left(\lambda_{31}-\lambda_{64}\right) .
$$

The problem of the solid body in a fluid in the case of Lyapunov-Steklov is a particular case of this metric (see subsection 4.2). The extra invariant $H_{4}$ is quadratic and the flow linearizes on 2-dimensional hyperelliptic Jacobians. More precisely

$$
\left.\bigcap_{j=1}^{4}\left\{x \in \mathbb{C}^{6}: Q_{j}(x)=c_{j}\right\}=J a c \text { (hyperelliptic curve } \mathscr{C} \text { of genus } 2\right) \backslash \mathscr{D},
$$

where $\mathscr{D}$ is a divisor of genus 17 , which contains 4 translates of the $\Theta$-divisor in $J a c(\mathscr{C})$, each of which is isomorphic to $\mathscr{C}$. The hyperelliptic curve $\mathscr{C}$ is a double cover of the curve $\mathscr{C}_{0}$ (isomorphic to $\mathbb{P}^{1}(\mathbb{C})$ ) defined as

$$
\left.\left\{t_{1}: t_{2}: t_{3}: t_{4}\right] \in \mathbb{P}^{3}(\mathbb{C}) \text { such that } \sum t_{j} Q_{j} \text { has rank } 3\right\} \text {. }
$$

The periods of the motion are given by the periods of the hyperelliptic curve $\mathscr{C}$. When studying the differential systems in this case as well as the invariants via Kowalewski-Painlevé's analysis, it is advantageous to rewrite them in a simpler form in order to reduce the notations and thus avoid too much calculation, since it requires less variables (see [6] for more detail). We show that this geodesic flow $X_{1}$ and a commuting flow $X_{2}$ can be written respectively in the form

$$
\begin{aligned}
\dot{x}_{1} & =x_{2} x_{6}, \\
\dot{x}_{2} & =\frac{1}{2} x_{3}\left(x_{1}+x_{4}\right), \\
\dot{x}_{3} & =\frac{1}{2} x_{2}\left(x_{1}+x_{4}\right), \\
\dot{x}_{4} & =x_{3} x_{5}, \\
\dot{x}_{5} & =x_{3} x_{4}, \\
\dot{x}_{6} & =x_{1} x_{2},
\end{aligned}
$$

and

$$
\begin{aligned}
& \dot{x}_{1}=x_{5} x_{6}, \\
& \dot{x}_{2}=x_{3} x_{4}, \\
& \dot{x}_{3}=x_{2} x_{4}, \\
& \dot{x}_{4}=x_{5}\left(2 x_{3}-x_{6}\right), \\
& \dot{x}_{5}=x_{4}\left(2 x_{3}-x_{6}\right), \\
& \dot{x}_{6}=x_{1} x_{5} .
\end{aligned}
$$


The Hamiltonian structure being determined by the Poisson bracket :

$$
\{H, F\}=\left\langle\frac{\partial H}{\partial x}, J \frac{\partial F}{\partial x}\right\rangle=\sum_{i, j} J_{i j} \frac{\partial H}{\partial x_{i}} \frac{\partial F}{\partial x_{j}}
$$

where

$$
J=\left(\begin{array}{cccccc}
0 & x_{3} & x_{2} & 0 & 0 & 2 x_{2}-x_{5} \\
-x_{3} & 0 & 0 & 0 & 0 & 0 \\
-x_{2} & 0 & 0 & 0 & 0 & 0 \\
0 & 0 & 0 & 0 & 0 & x_{5} \\
0 & 0 & 0 & 0 & 0 & x_{4} \\
-2 x_{2}+x_{5} & 0 & 0 & -x_{5} & -x_{4} & 0
\end{array}\right)
$$

The problem is to show, among other things, that the geodesic flow on $S O(4)$ for a left invariant in the case of the second metric is a weight homogeneous algebraic complete integrable system. Using the asymptotic expansion method as in the previous case (Kowalewski-Painlevé's analysis), we obtain the following results [6] :

Theorem 5.3. This geodesic flow has four quadric invariants :

$$
\begin{aligned}
& H_{1}=-x_{4}^{2}+x_{5}^{2}=c_{1}, \\
& H_{2}=-x_{1}^{2}+x_{6}^{2}=c_{2}, \\
& H_{3}=-x_{2}^{2}-x_{3}^{2}=\frac{c_{3}}{4}, \\
& H_{4}=-\left(x_{1}-x_{4}\right)^{2}+2\left(x_{2}-x_{5}\right)^{2}+2\left(x_{3}-x_{6}\right)^{2}=4 c_{4},
\end{aligned}
$$

(with generic $\left.\left(c_{1}, c_{2}, c_{3}, c_{4}\right) \in \mathbb{C}^{4}\right)$, and it evolues on some hyperelliptic Jacobians. The hyperelliptic curve is a double cover of the curve of rank four quadrics (isomorphic to $\mathbb{P}^{1}$ ) :

$$
\left\{t \in \mathbb{P}^{3} \text { such that } t_{1}\left(H_{1}-c_{1} x_{0}^{2}\right)+t_{2}\left(H_{2}-c_{2} x_{0}^{2}\right)+t_{3}\left(H_{3}-\frac{c_{3}}{4} x_{0}^{2}\right)+t_{4}\left(H_{4}-4 c_{4} x_{0}^{2}\right) \text { has rank } 4\right\},
$$

ramified at the six points where the rank drops to 3. The system in question possesses Laurent solutions depending on 5 free parameters and the affine surface defined by the constants of motion can be completed into a torus $T$ by adjoining a singular divisor $\mathscr{D}$. The latter consists of four copies $\mathscr{H}_{1}, \ldots, \mathscr{H}_{4}$ of the genus two hyperelliptic curves. Analyze the points of intersection of these curves. All these curves are translates of the $\Theta$-divisor by $\frac{1}{2}$-periods and three of these curves form a very ample and projectively normal divisor which results in the embedding of the Jacobian in $\mathbb{P}^{8}$ and the functions having poles there form a closed system of quadratic equations under differentiations as well as their ratios. The line bundle [DD] defines a polarization of type $(4,4)$ on $T$ and leads to an embedding in $\mathbb{P}^{15}$. The three flows $X_{1}, X_{2}$ and $2 X_{1}-X_{2}$ are doubly tangent to each of the four curves $\mathscr{H}_{1}, \ldots, \mathscr{H}_{4}$ at four points $P_{1}, \ldots, P_{4}$ and the sixteen half-periods on the torus are given by the total set of branch points of these hyperelliptic curves. The values of the constants of motion $c_{1} / c_{4}, c_{2} / c_{4}, c_{3} / c_{4}$ provide the three moduli for the full family of 2-dimensional hyperelliptic Jacobians.

\subsection{Geodesic flow on $S O(4)$ with a quartic invariant}

The form $H$ satisfies

$$
\left(\mu_{1}^{4}, \mu_{2}^{4}, \mu_{3}^{4}\right)=\lambda_{13} \lambda_{46} \lambda_{21} \lambda_{54} \lambda_{32} \lambda_{65}\left(\frac{1}{\lambda_{32} \lambda_{65}}, \frac{1}{\lambda_{13} \lambda_{46}}, \frac{1}{\lambda_{21} \lambda_{54}}\right) \text {. }
$$

The quantities $\zeta, \xi$ and $\eta$ defined by

$$
\zeta^{2} \equiv \frac{\lambda_{46}}{\lambda_{13}}, \quad \xi^{2} \equiv \frac{\lambda_{54}}{\lambda_{21}}, \quad \eta^{2} \equiv \frac{\lambda_{65}}{\lambda_{32}}
$$

satisfy the quadratic relations

$$
\zeta \xi+\xi \eta+\eta \zeta+1=0, \quad 3 \xi \eta+\eta-\xi+1=0 .
$$

The geodesic flow has a quartic invariant, evolves on Abelian surfaces $\widetilde{A} \subseteq \mathbb{P}^{23}(\mathbb{C})$ having period matrix

$$
\left(\begin{array}{llll}
2 & 0 & a & c \\
0 & 12 & c & b
\end{array}\right), \quad \operatorname{Im}\left(\begin{array}{ll}
a & c \\
c & b
\end{array}\right)>0, \quad(a, b, c \in \mathbb{C}),
$$


and it will be expressed in terms of Abelian integrals. See for details $[3,6]$. After the following linear change of coordinates, which is meaningful insofar $a \neq 0,-1,1,-1 / 3,1 / 3$,

$$
\begin{aligned}
& \left(\begin{array}{l}
x_{1} \\
x_{4}
\end{array}\right)=\sqrt{-1}\left(\begin{array}{cc}
a-1 & -1 \\
3 a+1 & 1
\end{array}\right)\left(\begin{array}{c}
(a-1) z_{1} \\
(3 a-1)(a+1) z_{4}
\end{array}\right), \\
& \left(\begin{array}{l}
x_{2} \\
x_{5}
\end{array}\right)=-\sqrt{-1}\left(\begin{array}{cc}
a+1 & -1 \\
3 a-1 & 1
\end{array}\right)\left(\begin{array}{c}
(a+1) z_{2} \\
(3 a+1)(a-1) z_{5}
\end{array}\right), \\
& \left(\begin{array}{l}
x_{3} \\
x_{6}
\end{array}\right)=\sqrt{-1}\left(\begin{array}{cc}
a-1 & a+1 \\
3 a+1 & 3 a-1
\end{array}\right)\left(\begin{array}{c}
(a-1) z_{3} \\
(a+1) z_{6}
\end{array}\right),
\end{aligned}
$$

the geodesic flow takes (after rescaling time) on the simple form :

$$
\begin{aligned}
& \dot{z}_{1}=z_{3} z_{5}, \\
& \dot{z}_{2}=z_{4} z_{6}, \\
& \dot{z}_{3}=\frac{1-a}{2} z_{4} z_{5}+z_{1} z_{5}+\frac{1+a}{2} z_{1} z_{2}, \\
& \dot{z}_{4}=\frac{2 a}{3 a-1} z_{5} z_{6}+\frac{a-1}{3 a-1} z_{2} z_{3}, \\
& \dot{z}_{5}=\frac{2 a}{3 a+1} z_{5} z_{6}+\frac{a+1}{3 a+1} z_{2} z_{3}, \\
& \dot{z}_{6}=\frac{1+a}{2} z_{4} z_{5}+z_{2} z_{4}+\frac{1-a}{2} z_{1} z_{2},
\end{aligned}
$$

with three quadratic invariants (in $z$ ) :

$$
\begin{aligned}
H_{1} & \equiv a F_{2}+\frac{1-a}{3 a+1} F_{7}=A_{1}, \\
H_{2} & \equiv-a F_{1}-\frac{a+1}{3 a-1}=A_{2}, \\
H_{3} & \equiv \frac{2 F_{6}}{(3 a-1)(3 a+1)}-\frac{F_{1}}{3 a+1}+\frac{F_{2}}{3 a-1}=A_{3},
\end{aligned}
$$

and a quartics invariant (in $z$ ) :

$$
\begin{aligned}
H_{4} \equiv-\frac{1-a}{3 a+1}\left(F_{1}^{2}+F_{4}^{2}\right)+\frac{1+a}{3 a-1}\left(F_{2}^{2}+F_{5}^{2}\right)+\frac{3\left(1-a^{2}\right)}{(3 a-1)(3 a+1)}\left(2 F_{1} F_{2}-F_{3}^{2}\right) \\
+\frac{4(1+a)}{(3 a-1)(3 a+1)} F_{2}\left(F_{6}+F_{8}\right)+\frac{4(1-a)}{(3 a-1)(3 a+1)} F_{1}\left(F_{6}+F_{7}\right)=A_{4},
\end{aligned}
$$

where

$$
\begin{aligned}
& F_{1}=z_{4}^{2}-z_{2} z_{5}, F_{2}=z_{5}^{2}-z_{1} z_{4}, F_{3}=z_{1} z_{2}-z_{4} z_{5}, F_{4}=\frac{2}{3 a-1}\left(z_{2} z_{3}-z_{5} z_{6}\right), \\
& F_{5}=\frac{-2}{3 a+1}\left(z_{1} z_{6}-z_{3} z_{4}\right), F_{6}=z_{1} z_{4}+z_{2} z_{5}-z_{3} z_{6}, F_{7}=z_{1}^{2}-z_{3}^{2}+z_{1} z_{4}, F_{8}=z_{2}^{2}-z_{6}^{2}+z_{2} z_{5} .
\end{aligned}
$$

The geodesic flow in question admits one family of Laurent solutions,

$$
z=\frac{\zeta}{t}\left(\mathbf{1}+U Y^{1} t+\frac{1}{\gamma Z^{2}+\delta}\left(U^{2} Y_{0}^{2}+\sum_{i=1}^{3} A_{i} Y_{i}^{2}\right) t^{2}+o\left(t^{3}\right)\right)
$$

where $\mathbf{1}=(1,1, \ldots, 1)^{\top}, Y^{1}, Y_{0}^{2}, Y_{i}^{2}$ are appropriate vectors depending on $Y, Z$ and $a$ only, $\gamma \equiv 4 a, \delta \equiv(a-1)(3 a+1)$ and

$$
\zeta=\operatorname{diag}\left(\frac{Y^{2}}{Z}, \frac{Z^{2}}{Y},-\frac{Y}{Z}, Z, Y,-\frac{Z}{Y}\right),
$$

with $Y, Z \in \mathbb{C}$ such that $Y^{2}+Z^{2}=1$. The 5-dimensional family of Laurent solutions depend on the parameters $Z, U, A_{1}, A_{2}$ and $A_{3}$. The vectors $Y_{i}^{2}$ can be chosen such that $H_{i}(z(t))=A_{i}$ for $i=1,2,3$. Confining the 5-dimensional family of Laurent solutions to the invariant manifold

$$
M_{c}=\bigcap_{i=1}^{4}\left\{z: H_{i}(z)=A_{i}\right\},
$$


yields a relation between the free parameters, defining a curve

$$
\mathscr{D}:\left\{\begin{array}{l}
(U, V, Y, Z) \text { such hat } Z^{2}=V, Y^{2}=1-V \text { and } \\
P(U, V)=\left(U^{2}(1-V) V(\alpha V+\beta)\right)^{2}-2 U^{2}(1-V) V P(V)+Q(V)=0,
\end{array}\right.
$$

where

$$
\alpha=16 a^{3}, \beta=(a-1)^{3}(3 a+1),
$$

and

$$
\begin{aligned}
P(V)= & (\alpha V+\beta)\left[\left(\left(3 a^{2}+1\right) A_{3}-A_{1}-A_{2}\right)(V-1)+A_{1} V-A_{2}(V-1)\right] \\
& -2 V(V-1)\left[A_{1}(1-a)^{3}(1+3 a)+A_{2}\left(1+a^{3}(1-3 a)-A_{3}\left(1-a^{2}\right)\left(1-9 a^{2}\right)\right],\right. \\
Q(V)= & {\left[\left(\left(3 a^{2}+1\right) A_{3}-A_{1}-A_{2}\right) V(V-1)-A_{1} V+A_{2}(V-1)\right]^{2} } \\
& +V(V-1)\left[(4 a V+(a-1)(3 a+1)) A_{4}+4 A_{1} A_{2}-(a-1)(3 a+1)(a+1)(3 a-1) A_{3}^{2}\right] .
\end{aligned}
$$

Note that

$$
P^{2}(V)-(\alpha V+\beta)^{2} Q(V)=V(1-V) R(V),
$$

with $R(V)$ being a cubic polynomial. The curve $\mathscr{D}$ is an unramified $4-1$ cover of the curve

$$
\mathscr{C}: P(U, V)=0 .
$$

In view of (5.16), the curve $\mathscr{C}$ itself is a double cover of the hyperelliptic curve

$$
\mathscr{H}: W^{2}=V(1-V) R(V),
$$

of genus 2, ramified over four points where $Q(V)=0$. Therefore $\mathscr{C}$ has genus 5 and $\mathscr{D}$ has genus 17 . The curve $\mathscr{D}$ must be thought of as being a very ample divisor on some Abelian surface $\widetilde{M}_{c}$, to be constructed according to the method described in the theorem 2.3. The curve $\mathscr{D}$, wrapped around $\widetilde{M}_{c}$ intersects itself transversally in 8 points, adding 8 to the genus 17 . Therefore the torus $\widetilde{M}_{c} \simeq \mathbb{C}^{2} / L_{\Omega}$ on which the geodesic flow linearizes, is defined by a period lattice $\Omega$ given by the columns of the matrix

$$
\Omega=\left(\begin{array}{cccc}
\delta_{1} & 0 & a & c \\
0 & \delta_{2} & c & b
\end{array}\right), \operatorname{Im}\left(\begin{array}{cc}
a & c \\
c & b
\end{array}\right)>0
$$

with

$$
\delta_{1} \delta_{2}=g(\mathscr{D})-1=24, \quad \delta_{1} \mid \delta_{2}, \quad \delta_{i} \in \mathbb{N}^{*} .
$$

So we have the following two possibilities : (i) $\delta_{1}=1, \delta_{2}=24$ and (ii) $\delta_{1}=2, \delta_{2}=12$. The line bundle

$$
L(\mathscr{D})=\left\{1, z_{1}, \ldots, z_{6}, f_{1}, \ldots, f_{5}, g_{1}, \ldots, g_{8}, h_{1}, \ldots, h_{4}\right\},
$$

is specified as follows

$$
\begin{aligned}
& g_{1}=-2 a z_{2} f_{2}-(1-a) z_{4} f_{3}, \quad g_{2}=-2 a z_{1} f_{1}-(1+a) z_{5} f_{3}, \quad g_{3}=(1-a) z_{5} f_{4}+(1+a) z_{4} f_{5}, \\
& g_{4}=(1+a) z_{5} f_{5}+(1-a) z_{1} f_{4}, \quad g_{5}=(1-a) z_{4} f_{4}+(1+a) z_{2} f_{5} \quad g_{6}=-(1-a) z_{3} f_{1}-(1+a) z_{6} f_{2}, \\
& g_{7}=2 a z_{5} f_{2}-(1-a) z_{1} f_{3}, \quad g_{8}=-2 a z_{4} f_{1}-(1+a) z_{2} f_{3}, \quad h_{1}=4 a^{2} f_{1} f_{2}+\left(1-a^{2}\right) f_{3}, \\
& h_{2}=2 a^{2} f_{1} f_{5}-(1-a) f_{3} f_{4}, \quad h_{3}=-2 a^{2} f_{2} f_{4}-(1+a) f_{3} f_{5}, \quad h_{4}=2 a^{2} f_{4} f_{5}+f_{3}\left((1+a) f_{2}-(1-a) f_{1}\right) .
\end{aligned}
$$

Now the reflection about the origin on the Abelian surface amounts to flipping the time for each linear flow on it, but since the flow $\frac{d z}{d t_{1}}$ given by (5.14) is quadratic and since the other flow $\frac{d z}{d t_{2}}$ (commuting with the first) is quartic (as it derives from the quartic Hamiltonian (5.15), flipping the signs of $t_{1}$ and $t_{2}$ for each of the flows amounts to the flip $\left(z_{1}, \ldots, z_{6}\right) \longmapsto\left(-z_{1}, \ldots,-z_{6}\right)$. 
From formula (5.10), the above line bundle $L(\mathscr{D})$ has in case $(i), 11$ even sections, 13 odd ones and in case (ii), 10 even sections, 14 odd ones, showing that case $(i i)$ is the only alternative and the period matrix has the form

$$
\left(\begin{array}{cccc}
2 & 0 & a & c \\
0 & 12 & c & b
\end{array}\right), \operatorname{Im}\left(\begin{array}{cc}
a & c \\
c & b
\end{array}\right)>0
$$

Differentiating $\frac{1}{z_{1}}$ and $\frac{z_{2}}{z_{1}}$ with respect to $t_{1}$ (corresponding to the flow (5.14)) and $t_{2}$ (corresponding to the quartic flow generated by $H_{4}(5.15)$ ), yield two differentials $\omega_{1}$ and $\omega_{2}$ defined on the curve $\mathscr{C}$ :

$$
\omega_{1}=\frac{\varphi(V) d V}{U \sqrt{V(1-V) R(V)}} \omega_{2}=\frac{d V}{U \sqrt{V(1-V) R(V)}},
$$

where $\varphi(V)$ is a rational function in $V$ having the form

$$
\left.\varphi(V)=\frac{4 a V+(a-1)(3 a+1)}{V(1-V)}\left[(\alpha V+\beta) U^{2} V(1-V)+\left(A_{3}\left(3 a^{2}+1\right)-A_{1}-A_{2}\right)(1-V) V-A_{1} V-A_{2}(1-V)\right)\right] .
$$

The restriction of the differentials $d t_{1}$ and $d t_{2}$ to the curve $\mathscr{D}$ are

$$
\omega_{1}=\left.d t_{1}\right|_{\mathscr{D}}=\varphi\left(Z^{2}\right) \omega_{2}, \omega_{2}=\left.d t_{2}\right|_{\mathscr{D}}=\frac{d Z}{U Y \sqrt{R}} .
$$

Recall that $\mathscr{C}$ is a double ramified cover of a hyperelliptic curve $\mathscr{H}$ of genus 2 , whose sheets are interchanged by the involution $(V, U) \longmapsto(V,-U)$. Hence

$$
\operatorname{Jac}(\mathscr{C})=\operatorname{Prym}(\mathscr{C} / \mathscr{H}) \oplus \operatorname{Jac}(\mathscr{H}) .
$$

Since $\omega_{1}$ and $\omega_{2}$ are both odd differentials for that involution, the flows evolve on the 3-dimensional Prym $(\mathscr{C} / \mathscr{H})$ and therefore $\widetilde{M}_{c} \subset \operatorname{Prym}(\mathscr{C} / \mathscr{H})$. This shows that $\operatorname{Prym}(\mathscr{C} / \mathscr{H})$ splits further, up to isogenies, into an elliptic curve $\mathscr{E}$ and the 2-dimensional invariant torus $\widetilde{M}_{c}$ :

$$
\widetilde{M}_{c} \oplus \mathscr{E}=\operatorname{Prym}(\mathscr{C} / \mathscr{H}) .
$$

In summary, we have

Theorem 5.4. The affine invariant surface $M_{c}$ for the Adler-van Moerbeke geodesic flow completes into a generic Abelian surface $\widetilde{M}_{c}$ of polarization $(1,6)$, i.e., defined by a period matrix of the form

$$
\left(\begin{array}{llll}
1 & 0 & a & c \\
0 & 6 & c & b
\end{array}\right), \quad \operatorname{Im}\left(\begin{array}{cc}
a & c \\
c & b
\end{array}\right)>0
$$

by adjoining at infinity a curve of genus 25 , with 8 normal crossings and smooth version $\mathscr{D}$. There exists an elliptic curve $\mathscr{E}$ such that $\widetilde{M}_{c}$ satisfies $\widetilde{M}_{c} \oplus \mathscr{E}=\operatorname{Prym}(\mathscr{C} / \mathscr{H})$. More precisely

$$
\bigcap_{j=1}^{4}\left\{x \in \mathbb{C}^{6}: H_{j}(x)=c_{j}\right\}=\widetilde{M}_{c} \backslash\{\text { a curve of genus } 25 \text { with } 8 \text { singular points }\} .
$$

Put in a more geometrical language, the tori $\widetilde{M}_{c}$ contain a very ample and projectively normal curve of geometric genus 25 , with 8 normal crossings whose smooth version $\mathscr{D}$ is a $4-1$ unramified cover of a curve $\mathscr{C}$ of genus 5 . The curve $\mathscr{C}$ itself is a double cover ramified over 4 points of a genus 2 hyperelliptic curve $\mathscr{H}$. Moreover, the linearization takes place on a 2-dimensional subtorus of the 3-dimensional Prym variety Prym $(\mathscr{C} / \mathscr{H})$ with

$$
\operatorname{Prym}(\mathscr{C} / \mathscr{H})=\widetilde{A} \oplus \mathscr{E},
$$

where $\mathscr{E}$ is an elliptic curve.

This situation provides a full description of the moduli for the Abelian surfaces of polarization $(1,6)$. For more information, see [6]. 


\section{The algebraic complete integrability of geodesic flow on $S O(n), n \geq 5$}

We have seen previously that if a system is algebraically completely integrable, then it must admit a family of meromorphic Laurent series depending on "dim(phase space) - 1" free parameters. Now, trying to generalize the result to the geodesic flow on $S O(n)$ for $n \geq 5$ using the same method leads to insurmountable calculations even with the help of a computer. Therefore, to answer the question concerning the classification of the metrics for which the geodesic flow on $S O(n)$ while taking into account these difficulties, it was necessary to resonate differently.

The geodesic flow for this problem takes the following commutator form (Euler-Arnold equations) :

$$
\dot{X}=[X, \Lambda . X],
$$

where

$$
X=\left(x_{i j}\right) \equiv \sum_{i<j} x_{i j} e_{i j} \in \operatorname{so}(n),
$$

and $\Lambda . X=\left(\lambda_{i j} X_{i j}\right), \lambda_{i j}=\lambda_{j i}$. The quadratic form $H$ is diagonal with regard to the customary $s o(n)$ coordinates (Manakov metric [29]), with

$$
\Lambda_{j k}=\frac{\beta_{j}-\beta_{k}}{\alpha_{j}-\alpha_{k}} \Longleftrightarrow[X, \beta]+[\alpha, \Lambda . X]=0, \forall X \in \operatorname{so}(n),
$$

with

$$
\alpha=\operatorname{diag}\left(\alpha_{1}, \ldots, \alpha_{n}\right), \quad \beta=\operatorname{diag}\left(\beta_{1}, \ldots, \beta_{n}\right), \quad \prod_{i<j}\left(\alpha_{i}-\beta_{j}\right) \neq 0,
$$

all $\Lambda_{j k}$ distinct. Note that it turns out that the geodesic flow on $S O(n)$ admits a lot of invariant manifolds on which they reduce to geodesic flow on $S O(3)$ and the solutions of the differential equation with initial conditions on these manifolds are elliptic functions and this without any condition on the metric. Haine [14] has shown that looking at solutions near these special a priori known solutions and imposing these solutions to be single-valued functions of $t \in \mathbb{C}$, suffices to single out the left invariant diagonal metrics for which the geodesic flow is algebraically completely integrable. Under the non-degeneracy assumption on the diagonal metric $\Lambda$ that all $\Lambda_{i j}$ be distinct, the system (6.1) is algebraically completely integrable with Abelian functions $x_{i j}$ if and only if the metric $\Lambda$ satisfies (6.2) (Manakov's conditions).

Theorem 6.1. For $n \geq 5$, Manakov's metrics are the only left invariant diagonal metrics on $S O(n)$ for which the geodesic flow is algebraically completely integrable.

This criterion was first used, without proof by Lyapunov [28] (the proof is due to Haine [14])), who showed that the only integrable tops whose solutions have analytic properties belong to the classical known cases : Euler top, Lagrange top and Kowalewski top.

\section{References}

[1] M. Adler, P. van Moerbeke, Linearization of Hamiltonian systems, Jacobi varieties and representation theory, Adv. in Math., 38 (1980), 318-379.

[2] M. Adler, P. van Moerbeke, The algebraic complete integrability of geodesic flow on SO(4), Invent. Math., 67 (1982), 297-331, with an appendix by D. Mumford.

[3] M. Adler, P. van Moerbeke, Algebraic completely integrable systems : a systematic approach, I, II, III, Séminaire de Mathématique, Rapport No 110, p.1-145, SC/MAPA - Institut de mathématique pure et appliquée, UCL, 1985.

[4] M. Adler, P. van Moerbeke, The Intersection of Four Quadrics in $\mathbb{P}^{6}$, Abelian Surfaces and their Moduli, Math. Ann., 279 (1987), 25-85.

[5] M. Adler, P. van Moerbeke, The complex geometry of the Kowalewski-Painlevé analysis, Invent. Math., 97 (1989), 3-51.

[6] M. Adler, P. van Moerbeke, P. Vanhaecke, Algebraic integrability, Painlevé geometry and Lie algebras, A series of modern surveys in mathematics, Volume 47, Springer-Verlag, 2004.

[7] V.I.: Arnold, Mathematical methods in classical mechanics, Springer-Verlag, Berlin-Heidelberg- New York, 1978, 2nd edn., Graduate Texts in Mathematics, 60, Springer-Verlag, New York, 1989. 
[8] A. Clebsch, Der Bewegung eines starren Körpers in einen Flüssigkeit, Math. Ann., 3 (1871), 238-268.

[9] R. Donagi, E. Markman, Spectral covers, algebraically completely integrable, hamiltonian systems, and moduli of bundles, Lecture Notes in Mathematics, 1620, Springer 1996.

[10] B.A. Dubrovin, Theta functions and non-linear equations, Russian Math. Surv., 36, 2 (1981), 11-92.

[11] L. Euler, Theoria motus corporum solidorum seu rigidorum, Rostock, 1765. Mémoires Acad. Sc, Berlin, 1758.

[12] P. A. Griffiths, J. Harris, Principles of algebraic geometry, Wiley-Interscience, New-York, 1978.

[13] L. Haine, Geodesic flow on SO(4) and Abelian surfaces, Math. Ann., 263 (1983), 435-472.

[14] L. Haine, The algebraic complete integrability of geodesic flow on $S O(N)$ and Abelian surfaces, Comm. Math. Phys., 94(2) (1984), 271-287.

[15] N. Hitchin, Stable bundles and integrable systems, Duke Mathematical Journal, 54,1 (1994), 91-114.

[16] C. G. J. Jacobi, Vorlesungen über Dynamik, Königsberg lectures of 1842-1843, (reprinted by Chelsea Publishing Co., New York, 1969.

[17] G. Kirchoff, Vorlesungen über Mathematische Physik, Vol. 1, Mechanik. Teubner, Leipzig, 1876.

[18] H. Knörrer, Integrable Hamiltonsche Systeme und algebraische Geometrie, Jahresber. Deutsch. Math.- Verein., 88 (2) (1986), 82-103.

[19] F. Kötter, Uber die Bewegung eines festen Körpers in einer Flüssigkeit I, II, Journal für die reine und angewandte Mathematik, 109 (1892), 51-81, 89-111.

[20] F. Kötter, Die von Steklow und Lyapunov entdeckten intgralen Fälle der Bewegung eines Körpers in einen Flüssigkeit Sitzungsber, Königlich Preussische Akad. d. Wiss., Berlin 6, 79-87 (1900).

[21] S. Kowalewski, Sur le problème de la rotation d'un corps solide autour d'un point fixe, Acta Math., 12 (1889), $177-232$.

[22] A. Lesfari, Geodesic flow on SO(4), Kac-Moody Lie algebra and singularities in the complex t-plane, Publ. Mat., Barc., 431 (1999), 261-279.

[23] A. Lesfari, The problem of the motion of a solid in an ideal fluid. Integration of the Clebsch's case, NoDEA, Nonlinear diff.Equ. Appl., 81 (2001), 1-13.

[24] A. Lesfari, Etude des solutions méromorphes d'équations différentielles, Ren. Semin. Mat. Univ. Politec. Torino, 654 (2007), 451-464.

[25] A. Lesfari, Prym varieties and applications, J. Geom. Phys., 58 (2008), 1063-1079.

[26] A. Lesfari, Algebraic integrability : the Adler-van Moerbeke approach, Regul. Chaotic Dyn., 16 (3-4) (2011), $187-209$.

[27] A. Lesfari, Introduction à la géométrie algébrique complexe, Éditions Hermann, Paris, 2015.

[28] A. Lyapunov, On a property of the differential equations of the problem of motion of a rigid body having a fixed point, Soobshceniya Kharkovskogo matematicheskog obshchestva (Transactions of the Kharkov Mathematical Society), Vol. IV (1894) and Reports of Kharkov. Math. Soc. Ser. 24, No 1-2 (1893), 81-85, Gesammelte Werke, Vol.1, $320-324$.

[29] S. V. Manakov, Remarks on the Integrals of the Euler Equations of the n-Dimensional Heavy Top, Fund. Anal. Appl., 10(4) (1976), 93-94.

[30] B.G. Moishezon, On n-dimensional compact varieties with n algebraically independent meromorphic functions, Amer. Math. Soc. Transl., 63 (1967), 51-177.

[31] J. Moser, Geometry of quadrics and spectral theory, Lecture delivred at the symposium in honor of S.S. Chern, Berkeley, 1979. Springer, Berlin, Heidelberg, New-York, 1980.

[32] D. Mumford, On the equations defining Abelian varieties I, II, III, Invent. Math., 1 (1966), 287-354, 3 (1967), 75-135, 3 (1967), 215-244.

[33] D. Mumford, Algebraic geometry I: complex projective varieties, Springer-Verlag, 1975.

[34] D. Mumford, Tata lectures on theta I, II, Progress in Math., Birkhaüser, Boston, 1983.

[35] P. Painlevé, Oeuvres, tomes 1,2,3. Édition du C.N.R.S., 1975.

[36] L. Poinsot, Théorie nouvelle de la rotation des corps, Journal de Liouville, Volume 16, (1851).

[37] V.A. Steklov, Über die Bewegung eines festen Körper in einer Flüssigkeit, Math. Ann., 42 (1893), 273-374.

[38] P. Vanhaecke, Integrable systems in the realm of algebraic geometry, Lecture Notes in Math., 1638, Springer-Verlag, Berlin, 2001. 